\title{
INCREASING PURSUER CAPTURABILITY BY USING HYBRID DYNAMICS
}

\author{
VALERY Y. GLIZER ${ }^{a}$, VlADIMIR TURETSKY $^{a, *}$ \\ ${ }^{a}$ Department of Applied Mathematics \\ Ort Braude College of Engineering, 51 Snunit Str., P.O.B. 78, Karmiel 2161002, Israel \\ e-mail: \{valery48, turet.sky1\}@braude.ac.i1
}

\begin{abstract}
A robust interception of a maneuverable target (evader) by an interceptor (pursuer) with hybrid dynamics is considered. The controls of the pursuer and the evader are bounded. The duration of the engagement is prescribed. The pursuer has two possible dynamic modes, which can be switched once during the engagement, while the dynamics of the evader are fixed. The case where for both dynamic modes there exists an unbounded capture zone was analyzed in our previous work. The conditions under which the pursuer can increase its capturability by utilizing the hybrid dynamics were established and the new robust capture zone was constructed. In the present paper, we extend this result to the cases where at least for one dynamic mode of the pursuer the capture zone is bounded. For these instances, conditions of increasing the pursuer's hybrid capturability are derived. Respective capture zones are constructed. Illustrative examples and results of extensive simulation for a realistic non-linear engagement model in the presence of a random wind are given.
\end{abstract}

Keywords: interception problem, hybrid interceptor's dynamics, robust capture zone.

\section{Introduction}

The interception problem is formulated for an engagement of two vehicles: an interceptor (pursuer) and a target (evader). In the simplified model, the dynamics of each vehicle are described by a first-order transfer function with time constants $\tau_{e}$ and $\tau_{p}$. The lateral acceleration commands of the players (controls) are bounded by the constants $a_{e}^{\max }$ and $a_{p}^{\max }$. Thus, the dynamics of each vehicle are completely described by the respective pair $\omega_{i}=\left(a_{i}^{\max }, \tau_{i}\right), i=e, p$. The interception problem admits two main mathematical formulations. If a target behavior can be predicted, this problem is modeled by an optimal control problem (see, e.g., the works of Glizer (1996; 1997) and the references therein). If a target behavior is unpredictable, the interception problem should be formulated as a robust control problem (Glizer and Turetsky, 2012). An important type of robust control problem is a differential game (see, e.g., Isaacs, 1965; Ho et al., 1965; Leitmann, 1980; Glizer, 1999; Turetsky and Shinar, 2003; Patsko and Turova, 2004; Shinar et al., 2013). Note that an unpredictable target maneuver can be regarded as a fault in the interception. Therefore, robustness with respect to such a target maneuver actually

\footnotetext{
*Corresponding author
}

is fault tolerance (Noura et al., 2009) in the interception.

The interception problem is frequently modeled by a planar linear pursuit-evasion differential game with a given duration, bounded controls, fixed players' dynamics and a cost function in the form of the miss distance. The solution of such a game is well known (Gutman and Leitmann, 1976; Shinar, 1981; Shima and Shinar, 2002; Gutman, 2006; Shinar et al., 2013). The key elements of this solution are (i) a scalarization of the original game and (ii) a decomposition of the space of the new (scalar) game into two regions, regular and singular. The regular region of the game space is completely covered by a family of candidate optimal trajectories. In this region, the optimal strategies of both players are of a "bang-bang" type and the value of the game depends on the initial conditions. In the singular region, which is the complement to the regular region in the game space, the optimal strategies of both players are arbitrary admissible and the game value is constant. The game space decomposition is completely determined by the pair $\left(\omega_{e}, \omega_{p}\right)$. Note that, if the game value in the singular region is zero, the singular region closure becomes the maximal robust capture zone, i.e., the set of all initial positions from which the zero miss distance (capture) is guaranteed by the optimal pursuer strategy against an arbitrary admissible evader control. 
Recently, interception problem formulations have been extended by assuming the hybrid dynamics of the opponents (see, e.g., Shinar et al., 2009; 2010; 2012; 2014; Poveda et al., 2012). This is motivated by the fact that modern intercepting vehicles can be equipped by guidance control systems of two types: aerodynamic control (in the atmosphere), and the thrust vector control (both in the space and in the atmosphere). The first type provides relatively large acceleration but is more inertial (larger values of $a_{p}^{\max }$ and $\tau_{p}$ ), while in the second type the values of $a_{p}^{\max }$ and $\tau_{p}$ are smaller. The switch from one type of the guidance system to the other during the interception makes the dynamics hybrid.

Hybrid dynamics systems arise naturally in various real-life applications (see, e.g., Lin and Antsaklis, 2003; Lygeros et al., 1999). Control problems with a single decision maker (Bartolini and Zolezzi, 1986; Chen and Fukuda, 1997; Utkin, 1983) as well as hybrid dynamics games (Gao et al., 2007; Mitchell et al., 2005; Shinar et al., 2009; 2010; 2012) have been studied. Shinar et al., 2009; 2010; 2012) consider the hybrid pursuit-evasion game separately with the hybrid pursuer and the hybrid evader.

In this paper, the robust interception problem with the pursuer's hybrid dynamics is treated. It is assumed that the pursuer can switch from the dynamic mode $\omega_{p 1}$ to $\omega_{p 2}$ once during the engagement. Note that by Shinar et al. (2009) it is assumed that in both pairs $\left(\omega_{e}, \omega_{p 1}\right)$ and $\left(\omega_{e}, \omega_{p 2}\right)$ the pursuer has the advantage in maneuverability, as well as in agility. Subject to these conditions, in the work of Shinar et al. (2009), the extended pursuer strategy, consisting of the control strategy and the dynamic schedule (order of the dynamic modes and the switch moment), was constructed. It was shown that its capture zone is larger than both maximal capture zones of a fixed dynamics game strategy. The objective of the present paper is searching some additional cases, where the condition of the pursuer's advantage both in the maneuverability and the agility is relaxed, while its hybrid dynamics increase the capture zone.

The paper is organized as follows. In Section 2, the problem is formulated rigorously. Its preliminary analysis leading to formulation of an auxiliary differential game, is presented in Section 3. In Section 4, this auxiliary game is solved. Section 5 is devoted to the main results of the paper. Simulation results for realistic engagement conditions are presented in Section 6. Concluding remarks are placed in Section 7. Some technical proofs are placed in Appendices.

\section{Problem formulation}

2.1. Engagement model. The engagement between two moving vehicles-an interceptor (pursuer) and a target (evader) — is considered. The mathematical model of this scenario is based on the following assumptions: (i) the engagement takes place in a horizontal plane, (ii) both the players have constant velocities and bounded lateral accelerations, (iii) the dynamics of each player is expressed by a first-order transfer function, (iv) the relative trajectory can be linearized with respect to the nominal collision geometry.

In Fig. 11 the schematic engagement geometry is depicted. The points $\left(x_{p}, y_{p}\right)$ and $\left(x_{e}, y_{e}\right)$ are current coordinates of the pursuer and the evader, respectively, $a_{p}$, $a_{e}$ are their lateral accelerations, $\varphi_{p}, \varphi_{e}$ are the respective angles between the velocity vectors and the $x$-axis (initial line of sight).

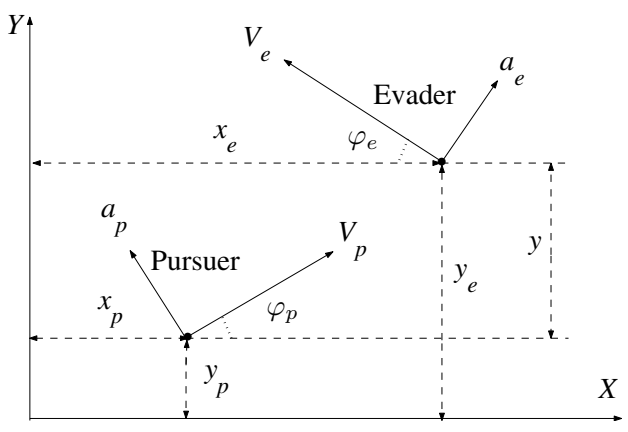

Fig. 1. Interception geometry.

Based on the small angles assumption (Shinar, 1981; Shima and Shinar, 2002; Glizer et al., 2012; Shinar et al., 2013), the trajectories of the pursuer and the evader can be linearized with respect to the nominal collision geometry, leading to a constant closing velocity $V_{c}$. The final interception time $t_{f}$ can be easily calculated for any given initial range $r_{0}: t_{f}=r_{0} / V_{c}$. This leads to the following linear model for $0 \leq t \leq t_{f}$ :

$$
\begin{array}{ll}
\dot{x}_{1}=x_{2}, & x_{1}(0)=0, \\
\dot{x}_{2}=x_{3}-x_{4}, & x_{2}(0)=x_{20}, \\
\dot{x}_{3}=\left(a_{e}^{\max } v-x_{3}\right) / \tau_{e}, & x_{3}(0)=0, \\
\dot{x}_{4}=\left(a_{p}^{\max } u-x_{4}\right) / \tau_{p}, & x_{4}(0)=0,
\end{array}
$$

where $x_{1}=y_{e}-y_{p}$ is the relative separation normal to the initial line of sight, $x_{2}$ is the relative normal velocity, $x_{3}$ and $x_{4}$ are the lateral accelerations of the evader and the pursuer, respectively, both normal to the initial line of sight, $\tau_{e}, \tau_{p}$ are the respective time constants, $a_{e}^{\max }, a_{p}^{\max }$ are the respective maximal absolute values of the lateral accelerations; $x_{20}=V_{e} \varphi_{e}^{0}-V_{p} \varphi_{p}^{0}, \varphi_{e}^{0}$ and $\varphi_{p}^{0}$ are initial values of $\varphi_{e}$ and $\varphi_{p}$, respectively.

The controls $v$ and $u$ of the evader and the pursuer, respectively, are the players' acceleration commands in the $y$-direction, normalized by $a_{e}^{\max }$ and $a_{p}^{\max }$, i.e., $v=$ $a_{e}^{c} / a_{e}^{\max }, u=a_{p}^{c} / a_{p}^{\max }$, where $a_{e}^{c}, a_{p}^{c}$ are the actual acceleration commands. 
Thus, the controls satisfy the constraints

$$
|v(t)| \leq 1, \quad|u(t)| \leq 1, \quad 0 \leq t \leq t_{f} .
$$

The system (1) can be rewritten in a matrix form as

$$
\dot{x}=A x+b u+c v, \quad x(0)=x_{0},
$$

where $x^{T}=\left(x_{1}, x_{2}, x_{3}, x_{4}\right), x_{0}^{T}=\left(0, x_{20}, 0,0\right)$,

$$
\begin{gathered}
A=\left[\begin{array}{cccc}
0 & 1 & 0 & 0 \\
0 & 0 & 1 & -1 \\
0 & 0 & -1 / \tau_{e} & 0 \\
0 & 0 & 0 & -1 / \tau_{p}
\end{array}\right], \\
b^{T}=\left(0,0,0, a_{p}^{\max } / \tau_{p}\right), \quad c^{T}=\left(0,0, a_{e}^{\max } / \tau_{e}, 0\right),
\end{gathered}
$$

and the superscript $T$ denotes the transposition.

Remark 1. The engagement model (3) is completely determined by two pairs of parameters, $\omega_{e}=\left(a_{e}^{\max }, \tau_{e}\right)$ and $\omega_{p}=\left(a_{p}^{\max }, \tau_{p}\right)$.

Consider the differential game with the dynamics (3), the control constraints (2) and the performance index

$$
\mathcal{J}=\left|x_{1}\left(t_{f}\right)\right|
$$

Note that (6) is the miss distance. The objective of the pursuer is minimizing (6), while the evader tends to maximize this value, by means of feedback strategies $u(t, x)$ and $v(t, x)$, respectively. This is called the original fixed dynamics game (OFDG).

2.2. Problem scalarization. Let introduce the function

$$
Z(t)=Z\left(t ; \omega_{e}, \omega_{p}\right)=d^{T} \Phi\left(t_{f}, t ; \tau_{e}, \tau_{p}\right) x\left(t ; \omega_{e}, \omega_{p}\right),
$$

where $x\left(t ; \omega_{e}, \omega_{p}\right)$ is the solution of (3), $\Phi\left(t_{f}, t ; \tau_{e}, \tau_{p}\right)$ is the transition matrix of the homogeneous system $\dot{x}=A x$, $d^{T}=(1,0,0,0)$.

The value of the function $Z(t)$ has the following physical interpretation: If $u \equiv 0$ and $v \equiv 0$ on the interval $\left[t, t_{f}\right]$, then the miss distance $\left|x_{1}\left(t_{f}\right)\right|$ equals $|Z(t)|$. Therefore, this function is called the zero-effort miss distance (ZEM).

By introducing the new independent variable $\vartheta=$ $t_{f}-t$ (time-to-go) and using (7), the ZEM can be expressed explicitly as

$$
\begin{aligned}
Z(\vartheta)= & Z\left(\vartheta, \omega_{e}, \omega_{p}\right) \\
= & x_{1}\left(t_{f}-\vartheta\right)+\vartheta x_{2}\left(t_{f}-\vartheta\right) \\
& +\tau_{e}^{2} \Psi\left(\vartheta / \tau_{e}\right) x_{3}\left(t_{f}-\vartheta\right) \\
& -\tau_{p}^{2} \Psi\left(\vartheta / \tau_{p}\right) x_{4}\left(t_{f}-\vartheta\right),
\end{aligned}
$$

where

$$
\Psi(\xi) \triangleq \exp (-\xi)+\xi-1>0, \xi>0 .
$$

Using (1) and (8) yields the differential equation for $Z(\vartheta)$,

$$
Z^{\prime}=h\left(\vartheta, \omega_{p}\right) u-h\left(\vartheta, \omega_{e}\right) v
$$

where the derivative with respect to $\vartheta$ is denoted by the prime, $h(\vartheta, \omega)=\tau a^{\max } \Psi(\vartheta / \tau), \omega=\left(a^{\max }, \tau\right)$, and $\vartheta_{0}=t_{f}, Z\left(\vartheta_{0}\right)=z_{0}=\vartheta_{0} x_{20}$. Note that $Z(0)=$ $x_{1}\left(t_{f}\right)$, i.e., the performance index (6) can be rewritten as $\mathcal{J}=|Z(0)|$. This allows us to associate the OFDG with a scalar game with the dynamics

$$
z^{\prime}=h\left(\vartheta, \omega_{p}\right) u-h\left(\vartheta, \omega_{e}\right) v, \quad z\left(\vartheta_{0}\right)=z_{0},
$$

the performance index

$$
J=|z(0)|
$$

and the control constraints

$$
|v(\vartheta)| \leq 1, \quad|u(\vartheta)| \leq 1, \quad 0 \leq \vartheta \leq \vartheta_{0},
$$

where the pursuer and the evader controls $u(\vartheta)$ and $v(\vartheta)$ are actually $u\left(t_{f}-\vartheta\right)$ and $v\left(t_{f}-\vartheta\right)$ of the OFDG. The game (11)-13) is called the scalar fixed dynamics game (SFDG). In the next subsection, a brief description of the well-known SFDG solution (Shinar, 1981; Shima and Shinar, 2002) is presented.

2.3. SFDG solution. The solution of the SFDG is based on the decomposition of the game space $(\vartheta, z)$ into two regions of different strategies. In the first (singular) region $D_{0}$, the optimal control strategies $u^{0}(\vartheta, z)$ and $v^{0}(\vartheta, z)$ are arbitrary subject to (13), and the value of the game is constant (zero or positive). If this game value is zero, the closure $\operatorname{clo}\left(D_{0}\right)$ of the singular region becomes the maximal robust capture zone, i.e., the set of all initial positions, from which the pursuer can guarantee zero miss distance against any admissible evader strategy.

In the second (regular) region $D_{1}=R^{2} \backslash D_{0}$, the optimal strategies have a "bang-bang" structure:

$$
u^{0}(\vartheta, z)=v^{0}(\vartheta, z)=\operatorname{sign}(z(\vartheta)),
$$

and the value of the game is non-zero, depending on the initial conditions. Note that $D_{0}$ and $D_{1}$ are symmetrical with respect to the $\vartheta$ axis. Below, all cases where the maximal robust capture zone exists are presented.

2.3.1. Unbounded capture zone. In the case

$$
a_{p}^{\max }>a_{e}^{\max }, \quad \frac{a_{p}^{\max }}{\tau_{p}} \geq \frac{a_{e}^{\max }}{\tau_{e}},
$$

the singular region is

$$
\begin{aligned}
D_{0} & =D_{0}\left(\omega_{e}, \omega_{p}\right) \\
& =\left\{(\vartheta, z): \vartheta>0,|z|<z^{*}\left(\vartheta, 0, \omega_{e}, \omega_{p}\right)\right\},
\end{aligned}
$$


where

$$
\begin{gathered}
z^{*}\left(\vartheta, \bar{\vartheta}, \omega_{e}, \omega_{p}\right)=\int_{\bar{\vartheta}}^{\vartheta} H\left(\xi, \omega_{e}, \omega_{p}\right) \mathrm{d} \xi \\
H\left(\xi, \omega_{e}, \omega_{p}\right) \triangleq h\left(\xi, \omega_{p}\right)-h\left(\xi, \omega_{e}\right)>0, \quad \xi>0 .
\end{gathered}
$$

For any initial position $\left(\vartheta_{0}, z_{0}\right)$ the value of the game is given by

$$
\begin{aligned}
& J^{*} \\
& =J^{*}\left(\vartheta_{0}, z_{0}, \omega_{e}, \omega_{p}\right) \\
& = \begin{cases}0, & \left(\vartheta_{0}, z_{0}\right) \in D_{0}\left(\omega_{e}, \omega_{p}\right), \\
\left|z_{0}\right|-z^{*}\left(\vartheta_{0}, 0, \omega_{e}, \omega_{p}\right), & \left(\vartheta_{0}, z_{0}\right) \notin D_{0}\left(\omega_{e}, \omega_{p}\right) .\end{cases}
\end{aligned}
$$

Thus, in this case, $\operatorname{clo}\left(D_{0}\right)$ is the maximal robust capture zone. Note that there exist positions inside the capture zone for any $\vartheta_{0}>0$, or, in other words, for any $t_{f}>0$ ("unbounded capture zone"). An example of the decomposition (for $t_{f}=4 \mathrm{~s}, \tau_{e}=0.2 \mathrm{~s}, a_{e}^{\max }=50 \mathrm{~m} / \mathrm{s}^{2}$, $\left.\tau_{p}=0.15 \mathrm{~s}, a_{p}^{\max }=70 \mathrm{~m} / \mathrm{s}^{2}\right)$ is shown in Fig. 2

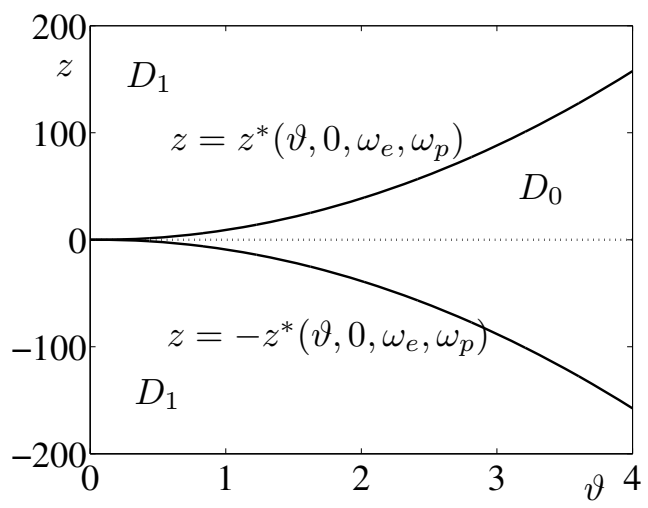

Fig. 2. Game space decomposition: an unbounded capture zone.

Remark 2. The vehicle maneuverability is determined by the value of its maximal lateral acceleration, while the vehicle agility is given by the ratio between its maximal lateral acceleration and its time constant. Thus, the condition (15) reads: "the interceptor is superior over the target both in maneuverability and agility".

\subsubsection{Bounded capture zone. In case}

$$
a_{p}^{\max }<a_{e}^{\max }, \quad \frac{a_{p}^{\max }}{\tau_{p}}>\frac{a_{e}^{\max }}{\tau_{e}},
$$

there exists $\vartheta_{c}>0$ such that the singular region is

$$
\begin{aligned}
D_{0}= & D_{0}\left(\omega_{e}, \omega_{p}\right) \\
= & \left\{(\vartheta, z): 0<\vartheta<\vartheta_{c},\right. \\
& \left.|z|<z^{*}\left(\vartheta, 0, \omega_{e}, \omega_{p}\right)\right\},
\end{aligned}
$$

and $z^{*}\left(\vartheta_{c}, 0, \omega_{e}, \omega_{p}\right)=0$.

The game value is given by 19 , i.e., $\operatorname{clo}\left(D_{0}\right)$ also is the (bounded) maximal robust capture zone. An example of the decomposition (for $t_{f}=8 \mathrm{~s}, \tau_{e}=0.5 \mathrm{~s}, a_{e}^{\max }=$ $100 \mathrm{~m} / \mathrm{s}^{2}, \tau_{p}=0.1 \mathrm{~s}, a_{p}^{\max }=90 \mathrm{~m} / \mathrm{s}^{2}$ ) is shown in Fig. 3

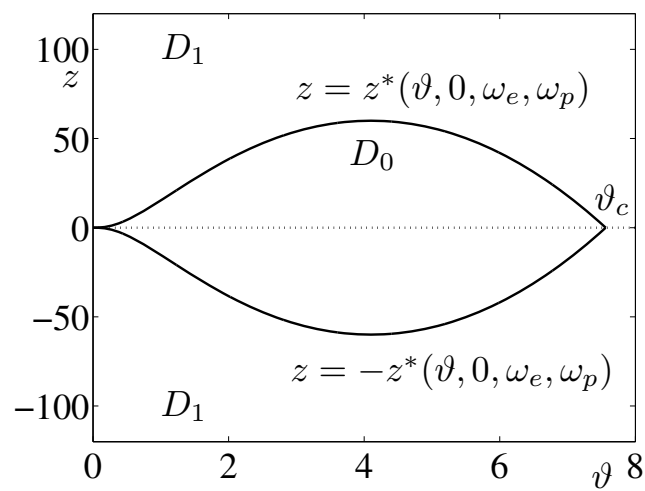

Fig. 3. Game space decomposition: a bounded capture zone.

2.4. Scalar hybrid robust capture problem. In what follows, the pair $\omega_{e}$ is assumed to be fixed. The pair $\omega_{p}$ is chosen by the pursuer from the prescribed set $\Omega_{p}=$ $\left\{\omega_{p 1}, \omega_{p 2}\right\}$ once during the engagement:

$$
\omega_{p}= \begin{cases}\omega_{p 1}, & \vartheta_{0} \geq \vartheta>\vartheta_{p}, \\ \omega_{p 2}, & \vartheta_{p} \geq \vartheta>0 .\end{cases}
$$

Thus, the pursuer has hybrid dynamics.

In the sequel, we assume that each of the pairs $\left(\omega_{e}, \omega_{p 1}\right)$ and $\left(\omega_{e}, \omega_{p 2}\right)$ satisfies one of the conditions 15 or 20], i.e., for each of the pursuer's dynamic modes, there exists the robust capture zone $\operatorname{clo}\left(D_{0}\right)$, unbounded or bounded. This assumption extends considerably the assumption of Shinar et al. (2009) that for both pursuer's dynamic modes there exists an unbounded capture zone.

Let $C_{0}\left(\omega_{e}, \omega_{p i}\right), i=1,2$, denote the pursuer's robust capture zone for the respective fixed dynamics. The objective of the paper is to find out in which case the pursuer can benefit from its hybrid dynamics, i.e., to increase its capturability. The latter means that by switching its dynamic mode and by choosing a proper control strategy the pursuer can guarantee the zero miss distance, robustly against any admissible evader behavior, from some set $\mathcal{C}_{p}$ of initial positions, satisfying

$$
\mathcal{C}_{p} \supset C_{0}\left(\omega_{e}, \omega_{p 1}\right) \cup C_{0}\left(\omega_{e}, \omega_{p 2}\right) .
$$

This problem is called the scalar hybrid robust capture problem (SHRCP). Thus, the objective of the paper is to distinguish the cases of SHRCP solvability.

Remark 3. In the case of hybrid dynamics, the physical meaning of the value (8) is no longer applicable. 
Nevertheless, for the sake of convenience, we continue to call it the ZEM.

Remark 4. In the case where both the pairs $\left(\omega_{e}, \omega_{p 1}\right)$ and $\left(\omega_{e}, \omega_{p 2}\right)$ satisfy the condition 15, the SHRCP is solved by Shinar et al. (2009). In this paper, the other cases are examined.

\section{Basic observations}

Although in the work of Shinar et al. (2009) it is assumed that for both the dynamic modes there exists an unbounded capture zone, the approach of this paper is rather general and can be applied in the other cases. This approach is based on the following basic observations.

3.1. Jump phenomenon. Due to $(8)$, for $\vartheta=\vartheta_{p}$, the ZEM has a jump

$$
\Delta Z\left(\vartheta_{p}\right)=\delta a_{p}\left(\vartheta_{p}\right)
$$

where

$$
\delta=\tau_{p 1}^{2} \Psi\left(\vartheta_{p} / \tau_{p 1}\right)-\tau_{p 2}^{2} \Psi\left(\vartheta_{p} / \tau_{p 2}\right),
$$

the function $\Psi(\xi)$ being given by (9). The graph of the ZEM with such a jump is shown in Fig. 4 for $t_{f}=5 \mathrm{~s}$, $z_{0}=1200 \mathrm{~m}, \vartheta_{p}=4.6 \mathrm{~s}$ and the data given in Table 1 with $j_{1}=1, j_{2}=2$. The players employ the strategies

$$
u(\vartheta, z)=v(\vartheta, z)=\operatorname{sign}(z(\vartheta)) .
$$

Table 1. Players' dynamics data: Set 1 .

\begin{tabular}{|c|c|c|}
\hline & Time constant $[\mathrm{s}]$ & Max. acc. command $\left[\mathrm{m} / \mathrm{s}^{2}\right]$ \\
\hline \hline Evader & $\tau_{e}=0.5$ & $a_{e}^{\max }=100$ \\
\hline \multirow{2}{*}{ Pursuer } & $\tau_{p j_{1}}=0.1$ & $a_{p j_{1}}^{\max }=150$ \\
\cline { 2 - 3 } & $\tau_{p j_{2}}=0.8$ & $a_{p j_{2}}^{\max }=240$ \\
\hline
\end{tabular}

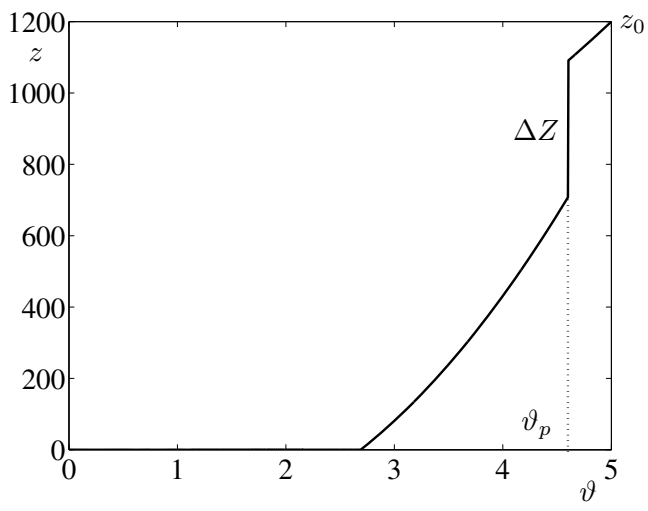

Fig. 4. Jump phenomenon.

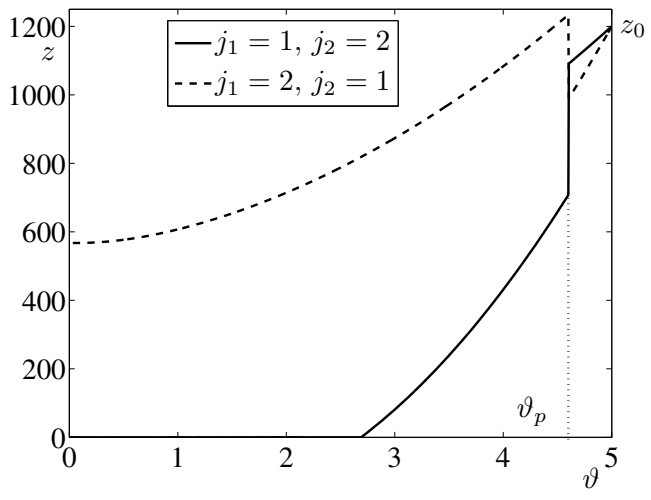

Fig. 5. Jump direction.

The jump is characterized by its direction (towards or outwards the $\vartheta$-axis) and magnitude.

It should be noted that, due to (11), a reasonable pursuer behavior is to $\operatorname{keep} \operatorname{sign}(u)=\operatorname{sign}(z)$. The latter, along with the fourth equation in (1), yields $\operatorname{sign}\left(a_{p}\right)=$ $\operatorname{sign}(z)$. In such a case, the jump direction with respect to the $\vartheta$-axis is completely determined by the following equality (Shinar et al., 2009, Lemma 3):

$$
\operatorname{sign}(\delta)=\operatorname{sign}\left(\tau_{p 1}-\tau_{p 2}\right) .
$$

In Fig. 5, the graphs of ZEM are depicted for $t_{f}=5 \mathrm{~s}$, $z_{0}=1200 \mathrm{~m}, \vartheta_{p}=4.6 \mathrm{~s}$, the strategies (26) and the data of Table 1 with different orders of dynamic modes. It is seen that in the first case $\left(j_{1}=1, j_{2}=2\right)$ the jump is directed towards the $\vartheta$-axis, because $\tau_{p 1}<\tau_{p 2}$, yielding $\delta<0$. In the second case $\left(j_{1}=2, j_{2}=1\right)$ the opposite jump direction is observed.

Due to (24) and (25), the jump magnitude depends both on the order of the pursuer dynamic modes and on the switch moment $\vartheta_{p}$. In Fig. 5, $|\Delta Z(4.6)|=383.71$ $\mathrm{m}$ for $j_{1}=1, j_{2}=2$, while $|\Delta Z(4.6)|=242.85 \mathrm{~m}$ for $j_{1}=2, j_{2}=1$. In Fig. 6, the dependence of the jump magnitude on the switch moment is shown for the data of Table 1 with $j_{1}=1, j_{2}=2$, for $t_{f}=5 \mathrm{~s}, z_{0}=1200 \mathrm{~m}$, for the strategies (26) and for different switch moments $\vartheta_{p}$. It is seen that, for different switch moments, the jump magnitudes differ. Namely, $|\Delta Z(4.6)|=383.71$ $\mathrm{m},|\Delta Z(4.1)|=337.74 \mathrm{~m},|\Delta Z(3.6)|=285.64 \mathrm{~m}$, $|\Delta Z(3.1)|=232.94 \mathrm{~m}$.

3.2. Requirements for pursuer dynamic modes. Since we assume that the initial interception position satisfies

$$
\left(\vartheta_{0}, z_{0}\right) \notin C_{0}\left(\omega_{e}, \omega_{p 1}\right) \cup C_{0}\left(\omega_{e}, \omega_{p 2}\right),
$$

the first pursuer dynamic mode for $\vartheta \in\left(\vartheta_{p}, \vartheta_{0}\right]$, along with the ZEM jump at $\vartheta=\vartheta_{p}$, should steer the ZEM into 


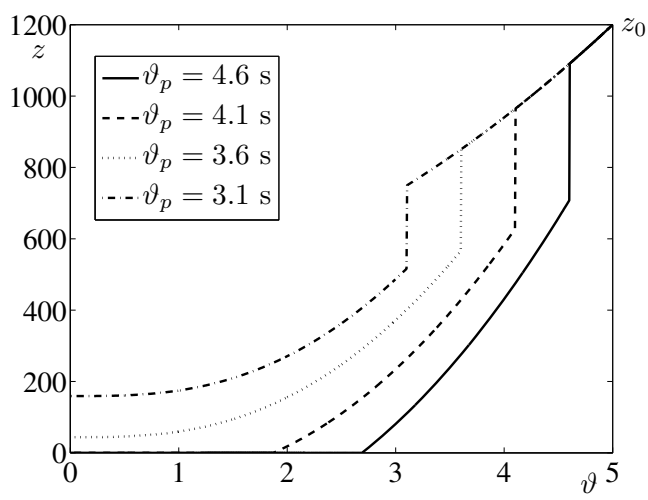

Fig. 6. Jump magnitude.

the capture zone $C_{0}\left(\omega_{e}, \omega_{p 2}\right)$ :

$$
\left(\vartheta_{p}, z\left(\vartheta_{p}+0\right)+\Delta Z\left(\vartheta_{p}\right)\right) \in C_{0}\left(\omega_{e}, \omega_{p 2}\right) .
$$

It is reasonable to require that this jump be directed towards the $\vartheta$-axis, i.e.,

$$
\delta<0
$$

This, due to 27, leads to the requirement

$$
\tau_{p 1}<\tau_{p 2} .
$$

In what follows, we assume that the condition (31) holds.

In Fig. 7, two ZEM trajectories are depicted for the data of Table 1 with $j_{1}=1, j_{2}=2$, for $\vartheta_{0}=5 \mathrm{~s}$, $z_{0}=1200 \mathrm{~m}$, for the strategies 26 and for two switch moments $\vartheta_{p}=4.6 \mathrm{~s}$ and $\vartheta_{p}=3.1 \mathrm{~s}$. For these data, both pairs $\left(\omega_{e}, \omega_{p 1}\right)$ and $\left(\omega_{e}, \omega_{p 2}\right)$ satisfy (15), i.e., both capture zones $C_{0}\left(\omega_{e}, \omega_{p 1}\right) \neq \emptyset$ and $C_{0}\left(\omega_{e}, \omega_{p 2}\right) \neq \emptyset$ are unbounded. The initial position $\left(\vartheta_{0}, z_{0}\right)$ satisfies (28). It is seen that for $\vartheta_{p}=4.6 \mathrm{~s}$ the inclusion (29) is satisfied, while for $\vartheta_{p}=3.1 \mathrm{~s}$, it is not.

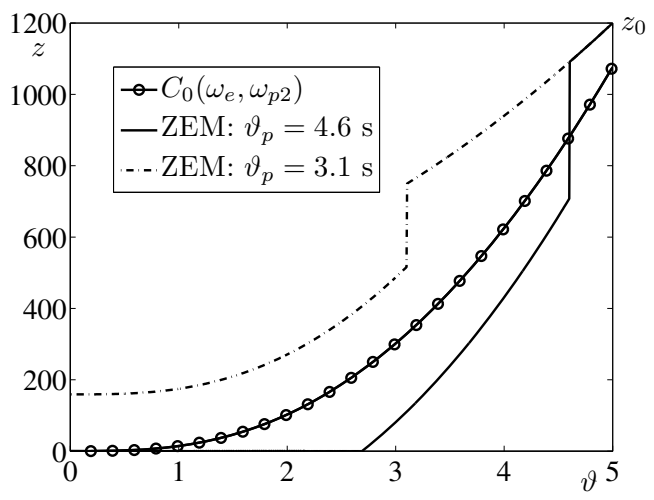

Fig. 7. Steering the ZEM to unbounded $C_{0}\left(\omega_{e}, \omega_{p 2}\right)$.

A similar effect is demonstrated in Fig. 8 for the data presented in Table 2 with $j_{1}=1, j_{2}=2$, for $\vartheta_{0}=1.9$ $\mathrm{s}, z_{0}=0.01 \mathrm{~m}$, for the strategies 26 and for two switch moments $\vartheta_{p}=1.85 \mathrm{~s}$ and $\vartheta_{p}=1.75 \mathrm{~s}$. For these data, both pairs $\left(\omega_{e}, \omega_{p 1}\right)$ and $\left(\omega_{e}, \omega_{p 2}\right)$ satisfy (20), i.e., both the capture zones $C_{0}\left(\omega_{e}, \omega_{p 1}\right) \neq \emptyset$ and $C_{0}\left(\omega_{e}, \omega_{p 2}\right) \neq \emptyset$ are bounded. The initial position $\left(\vartheta_{0}, z_{0}\right)$ satisfies (28). It is seen that for $\vartheta_{p}=1.85 \mathrm{~s}$ the inclusion (29) is satisfied, while for $\vartheta_{p}=1.75 \mathrm{~s}$ it is not.

Table 2. Players' dynamics data: Set 2.

\begin{tabular}{|c|c|c|}
\hline & Time constant $[\mathrm{s}]$ & Max. acc. command $\left[\mathrm{m} / \mathrm{s}^{2}\right]$ \\
\hline \hline Evader & $\tau_{e}=0.2$ & $a_{e}^{\max }=150$ \\
\hline \multirow{2}{*}{ Pursuer } & $\tau_{p j_{1}}=0.08$ & $a_{p j_{1}}^{\max }=120$ \\
\cline { 2 - 3 } & $\tau_{p j_{2}}=0.1$ & $a_{p j_{2}}^{\max }=135$ \\
\hline
\end{tabular}

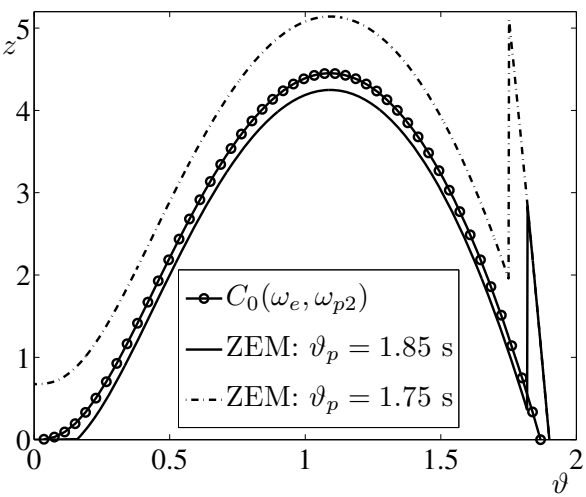

Fig. 8. Steering the ZEM to bounded $C_{0}\left(\omega_{e}, \omega_{p 2}\right)$.

3.3. Requirements for the pursuer strategy and the switch moment. In the examples presented in Figs. 7 and 8 the condition (29) is met for $v=\operatorname{sign}(z)$. However, it is extremely important that this condition be fulfilled robustly with respect to any admissible evader control $v$. This is guaranteed by an optimal pursuer strategy in the following auxiliary differential game of kind (ADGK). Consider the system

$$
\begin{aligned}
z^{\prime} & =h\left(\vartheta, \omega_{p 1}\right) u-h\left(\vartheta, \omega_{e}\right) v, & z\left(\vartheta_{0}\right) & =z_{0}, \\
a_{p}^{\prime} & =\left(a_{p}-a_{p 1}^{\max } u\right) / \tau_{p 1}, & a_{p}\left(\vartheta_{0}\right) & =0,
\end{aligned}
$$

where the controls satisfy the constraints

$$
|u(\vartheta)| \leq 1, \quad|v(\vartheta)| \leq 1, \quad \vartheta_{0} \geq \vartheta \geq \vartheta_{p} .
$$

The objective of the pursuer is to provide the inclusion (29) for all admissible evader controls $v$ by using a feedback strategy $u=u\left(\vartheta, z, a_{p}\right)$.

Remember that the original aim of the pursuer is not only guaranteeing $z(0)=0$, but making it from as wide a set of initial positions $\left(\vartheta_{0}, z_{0}\right)$, as possible i.e., from the capture zone $\mathcal{C}_{p}$. This means that among all 
strategies solving the ADGK (32)- (33), 29), the pursuer has to choose the one for which $\left|z_{0}\right|$ is maximal possible. This allows us to reformulate the ADGK as the differential game consisting of the dynamics (32), the constraints (33) and the performance index

$$
J_{\mathrm{aux}}=\left|z\left(\vartheta_{p}\right)+\Delta Z\left(\vartheta_{p}\right)\right|,
$$

to be minimized by the pursuer and maximized by the evader by using feedback strategies $u=u\left(\vartheta, z, a_{p}\right)$ and $v=v\left(\vartheta, z, a_{p}\right)$, respectively. This is called the auxiliary differential game of degree (ADGD).

For the above-mentioned maximal possible $\left|z_{0}\right|$, the ADGD optimal trajectory terminates at the point $\left(\vartheta_{p}, z\left(\vartheta_{p}\right)\right)$, which is brought by the jump $\Delta Z\left(\vartheta_{p}\right)$ onto the boundary of $C_{0}\left(\omega_{e}, \omega_{p 2}\right)$. Thus, due to (16) and (21), the requirement 29) becomes

$$
\left|z\left(\vartheta_{p}\right)+\Delta Z\left(\vartheta_{p}\right)\right|=z^{*}\left(\vartheta_{p}, 0, \omega_{e}, \omega_{p 2}\right),
$$

where the function $z^{*}$ is given by (17).

In Fig. 9, such an optimal trajectory is shown for the data of Table 1, $\vartheta_{p}=4.6 \mathrm{~s}$. This trajectory emanates from the initial position $\left(\vartheta_{0}=5 \mathrm{~s}, z_{0}=1375 \mathrm{~m}\right) \notin$ $C_{0}\left(\omega_{e}, \omega_{p 1}\right) \cup C_{0}\left(\omega_{e}, \omega_{p 2}\right)$ and terminates at the point $(4.6 \mathrm{~s}, 1266.5 \mathrm{~m})$. The latter, due to the jump, is brought onto the boundary of $C_{0}\left(\omega_{e}, \omega_{p 2}\right)$.

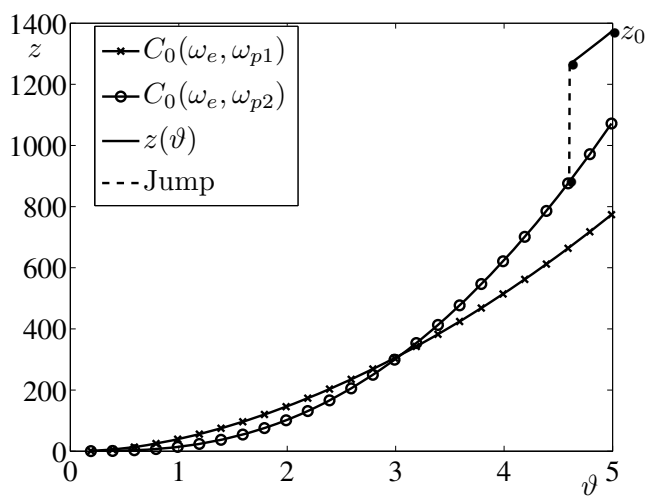

Fig. 9. Jump to boundary of $C_{0}\left(\omega_{e}, \omega_{p 2}\right)$.

At the final stage of constructing the capture zone $\mathcal{C}_{p}$, the pursuer can additionally increase $\left|z_{0}\right|$ by a proper choice of the switch moment $\vartheta_{p}$.

\section{Solution of the ADGD}

In the case where the pair $\left(\omega_{e}, \omega_{p 1}\right)$ satisfies the condition (15), the ADGD 32-34) is solved by Shinar et al. (2009). The solution was obtained based on scalarization of the differential game by the terminal projection transformation

$$
\begin{aligned}
w(\vartheta) & =w\left(\vartheta, z(\vartheta), a_{p}(\vartheta)\right) \\
& =d_{\vartheta_{p}}^{T} \Lambda\left(\vartheta_{p}, \vartheta\right)\left[\begin{array}{c}
z(\vartheta) \\
a_{p}(\vartheta)
\end{array}\right],
\end{aligned}
$$

where $d_{\vartheta_{p}}^{T}=[1, \delta]$ and $\Lambda\left(\vartheta, \vartheta_{0}\right)$ is the transition matrix of the homogeneous system, corresponding to $\underline{32}$, for $\tau_{p}=\tau_{p 1}$ :

$$
\Lambda\left(\vartheta, \vartheta_{0}\right)=\left[\begin{array}{cc}
1 & 0 \\
0 & \exp \left(\left(\vartheta-\vartheta_{0}\right) / \tau_{p 1}\right)
\end{array}\right]
$$

By virtue of (36),

$$
J_{\mathrm{aux}}=\left|w\left(\vartheta_{p}\right)\right| .
$$

Due to (36) and 37,

$$
w(\vartheta)=z(\vartheta)+\delta\left(\exp \left(\left(\vartheta_{p}-\vartheta\right) / \tau_{p 1}\right)\right) a_{p}(\vartheta) .
$$

By using (32) and (39), $w(\vartheta)$ satisfies the differential equation

$$
w^{\prime}=\hat{h}\left(\vartheta, \vartheta_{p}, \tau_{p 1}, \tau_{p 2}, a_{p 1}^{\max }\right) u-h\left(\vartheta, \omega_{e}\right) v
$$

and the initial condition

$$
w_{0} \triangleq w\left(\vartheta_{0}\right)=z_{0},
$$

where we have

$$
\begin{aligned}
& \hat{h}\left(\vartheta, \vartheta_{p}, \tau_{p 1}, \tau_{p 2}, a_{p 1}^{\max }\right) \\
& \quad=h\left(\vartheta, \omega_{p 1}\right)-\left(\delta a_{p 1}^{\max } / \tau_{p 1}\right) \exp \left(\left(\vartheta_{p}-\vartheta\right) / \tau_{p 1}\right) .
\end{aligned}
$$

Thus, the scalar auxiliary differential game has the dynamics (40) with the initial condition (41), the control constraints (33) and the cost function (38), to be minimized by the pursuer and maximized by the evader.

In the present paper, we solve this differential game in the cases where $\left(\omega_{e}, \omega_{p 1}\right)$ satisfies either (15) or 20). We propose here an approach to the solution different from the one by Shinar et al. (2009). Due to Glizer and Turetsky (2008), the solution is determined by the sign of $\hat{H}\left(\vartheta_{p}\right)$, where

$$
\begin{aligned}
\hat{H}(\vartheta)= & \hat{H}\left(\vartheta, \omega_{e}, \omega_{p 1}, \tau_{p 2}\right) \\
\triangleq & \hat{h}\left(\vartheta, \vartheta_{p}, \tau_{p 1}, \tau_{p 2}, a_{p 1}^{\max }\right)-h\left(\vartheta, \omega_{e}\right) \\
= & H\left(\vartheta, \omega_{e}, \omega_{p 1}\right) \\
& -\left(\delta a_{p 1}^{\max } / \tau_{p 1}\right) \exp \left(\left(\vartheta_{p}-\vartheta\right) / \tau_{p 1}\right) .
\end{aligned}
$$

The sign of this function, along with some additional conditions, yields the decomposition of the state strip $\hat{D}=\left\{(\vartheta, w): \vartheta \in\left[\vartheta_{p}, \vartheta_{0}\right], w \in \mathbb{R}\right\}$ into two regions: the regular region $\hat{D}_{1}$, completely covered by the optimal trajectories, and the singular region $\hat{D}_{0}=\hat{D} \backslash \hat{D}_{1}$. The regular region $\hat{D}_{1}$ is closed and is the invariant set of the system (40) for optimal strategies of both players.

In some cases the initial point $\left(\vartheta_{0}, w_{0}\right)$ belongs only to the regular region, while in the other cases it can belong to the regular as well as singular regions. 
Note that, if $\left(\omega_{e}, \omega_{p 1}\right)$ satisfies $[15)$, then $\hat{H}\left(\vartheta_{p}\right)>0$, while in the case (20) the sign of $\hat{H}\left(\vartheta_{p}\right)$ can be either positive or negative. Let

$$
w^{*}(\vartheta, \bar{\vartheta})=w^{*}\left(\vartheta, \bar{\vartheta} ; \omega_{e}, \omega_{p 1}, \tau_{p 2}\right) \triangleq \int_{\bar{\vartheta}}^{\vartheta} \hat{H}(\xi) \mathrm{d} \xi .
$$

\subsection{Case I: $\hat{H}\left(\vartheta_{p}\right) \geq 0$.}

4.1.1. Subcase I.1: $\boldsymbol{w}^{*}\left(\boldsymbol{\vartheta}, \boldsymbol{\vartheta}_{p}\right)>0, \forall \vartheta \in\left[\boldsymbol{\vartheta}_{p}, \boldsymbol{\vartheta}_{0}\right]$. In this subcase, the optimal strategies are

$$
\begin{aligned}
\hat{u}(\vartheta, w) & =\hat{v}(\vartheta, w) \\
& = \begin{cases}\operatorname{arbitrary} \text { s.t. [33), } & |w|<w^{*}\left(\vartheta, \vartheta_{p}\right), \\
\operatorname{sign}(w), & |w| \geq w^{*}\left(\vartheta, \vartheta_{p}\right) .\end{cases}
\end{aligned}
$$

The game value is

$$
\begin{aligned}
& J_{\text {aux }}^{*}\left(\vartheta_{0}, w_{0}\right) \\
& = \begin{cases}0, & \left|w_{0}\right|<w^{*}\left(\vartheta_{0}, \vartheta_{p}\right), \\
\left|w_{0}\right|-w^{*}\left(\vartheta_{0}, \vartheta_{p}\right), & \left|w_{0}\right| \geq w^{*}\left(\vartheta_{0}, \vartheta_{p}\right) .\end{cases}
\end{aligned}
$$

Note that the set

$$
\hat{D}_{0}=\left\{(\vartheta, w): \vartheta \in\left[\vartheta_{p}, \vartheta_{0}\right],|w|<w^{*}\left(\vartheta, \vartheta_{p}\right)\right\}
$$

is the singular region in the scalar auxiliary differential game. Its closure $\operatorname{clo}\left(\hat{D}_{0}\right)$ is the maximal robust capture zone in this game. In Fig. 10, the singular region is depicted for $\tau_{e}=0.5 \mathrm{~s}, a_{e}^{\max }=100 \mathrm{~m} / \mathrm{s}^{2}, \tau_{p 1}=0.1$ $\mathrm{s}, a_{p 1}^{\max }=150 \mathrm{~m} / \mathrm{s}^{2}, \tau_{p 2}=0.8, \vartheta_{0}=4 \mathrm{~s}, \vartheta_{p}=2 \mathrm{~s}$.

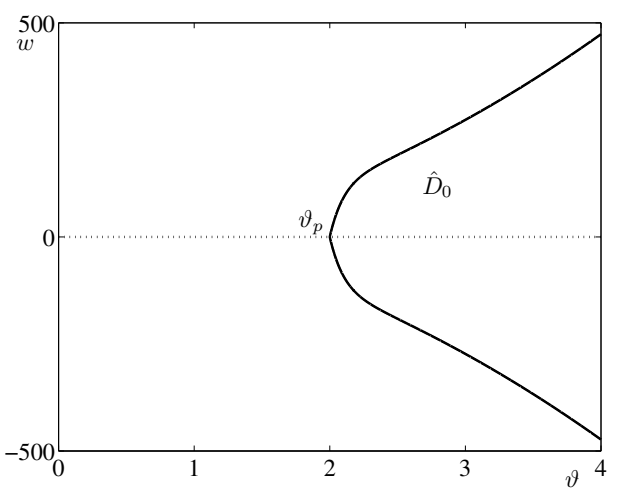

Fig. 10. Singular region in Case I.1: $\left(\omega_{e}, \omega_{p 1}\right)$ satisfies 15.

In Fig. 11, the singular region is depicted for the data presented in Table 3 and for $\vartheta_{p}=0.5 \mathrm{~s}, \vartheta_{0}=2 \mathrm{~s}$.
Table 3. Players' dynamics data: set 3 .

\begin{tabular}{|c|c|c|}
\hline & Time constant $[\mathrm{s}]$ & Max. acc. command $\left[\mathrm{m} / \mathrm{s}^{2}\right]$ \\
\hline \hline Evader & $\tau_{e}=0.2$ & $a_{e}^{\max }=50$ \\
\hline \multirow{2}{*}{ Pursuer } & $\tau_{p 1}=0.06$ & $a_{p 1}^{\max }=48$ \\
\cline { 2 - 3 } & $\tau_{p 2}=0.2$ & $a_{p 2}^{\max }=55$ \\
\hline
\end{tabular}

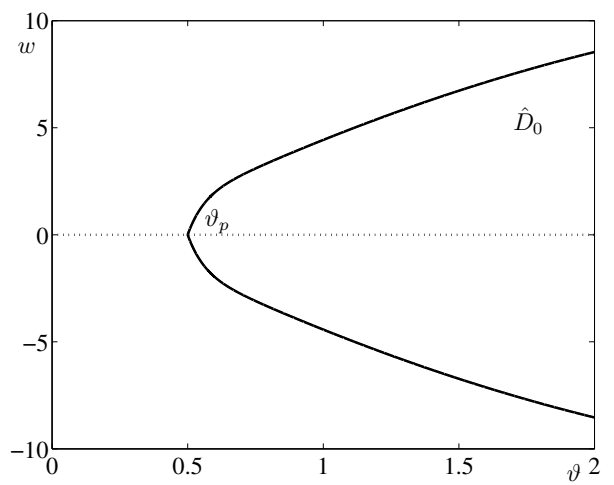

Fig. 11. Singular region in Case I.1: $\left(\omega_{e}, \omega_{p 1}\right)$ satisfies 20.

4.2. Subcase I.2: $\exists \vartheta \in\left(\vartheta_{p}, \vartheta_{0}\right): \boldsymbol{w}^{*}\left(\vartheta, \vartheta_{p}\right)<0$. Let

$$
\begin{aligned}
\vartheta_{1} \triangleq & \min \left\{\vartheta \in\left(\vartheta_{p}, \vartheta_{0}\right):\right. \\
& \left.w^{*}\left(\vartheta-0, \vartheta_{p}\right)>0, w^{*}\left(\vartheta+0, \vartheta_{p}\right)<0\right\} .
\end{aligned}
$$

In this subcase, the optimal strategies are

$$
\begin{aligned}
& \hat{u}(\vartheta, w) \\
& =\hat{v}(\vartheta, w) \\
& = \begin{cases}\operatorname{arbitrary} \text { s.t. }[33), & |w|<w^{*}\left(\vartheta, \vartheta_{p}\right), \vartheta_{p} \leq \vartheta<\vartheta_{1}, \\
\operatorname{sign}(w), & \text { otherwise. }\end{cases}
\end{aligned}
$$

The set

$$
\hat{D}_{0}=\left\{(\vartheta, w): \vartheta \in\left[\vartheta_{p}, \vartheta_{1}\right),|w|<w^{*}\left(\vartheta, \vartheta_{p}\right)\right\}
$$

is the singular region in the scalar auxiliary differential game. In this subcase, $\left(\vartheta_{0}, w_{0}\right) \notin \hat{D}_{0}$. The game value is

$$
J_{\text {aux }}^{*}\left(\vartheta_{0}, w_{0}\right)=\left|w_{0}\right|-w^{*}\left(\vartheta_{0}, \vartheta_{p}\right) .
$$

In Fig. 12, the singular region is depicted for the data presented in Table 3 and for $\vartheta_{p}=0.7 \mathrm{~s}, \vartheta_{0}=8 \mathrm{~s}$. In this example, $\vartheta_{1}=6.9 \mathrm{~s}$.

4.3. Case II: $\hat{\boldsymbol{H}}\left(\boldsymbol{\vartheta}_{\boldsymbol{p}}\right)<\boldsymbol{0}$. Due to (30) and (42), the value $\hat{H}\left(\vartheta_{p}\right)$ can be negative if and only if $H\left(\vartheta_{p}, \omega_{e}, \omega_{p 1}\right)<0$. By virtue of the SFDG solution, this inequality is fulfilled only for the pair $\left(\omega_{e}, \omega_{p 1}\right)$, satisfying 


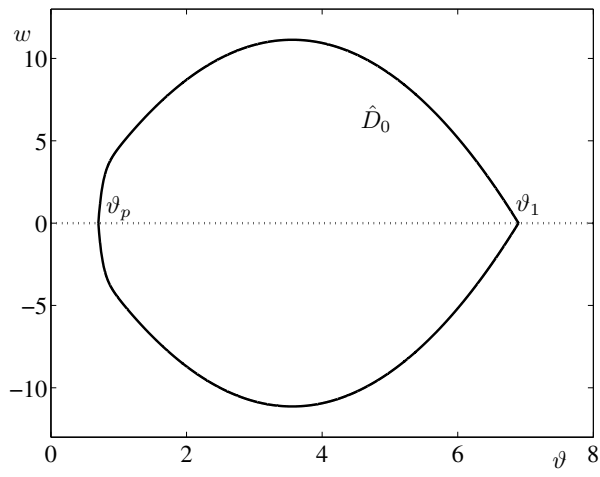

Fig. 12. Singular region in Case I.2: $\left(\omega_{e}, \omega_{p 1}\right)$ satisfies 20].

(20) (the capture zone $C_{0}\left(\left(\omega_{e}, \omega_{p 1}\right)\right.$ is bounded, $0 \leq \vartheta \leq$ $\left.\vartheta_{c 1}\right)$, and for

$$
\vartheta_{p}>\vartheta_{c 1} .
$$

Lemma 1. Let the pair $\left(\omega_{e}, \omega_{p 1}\right)$ satisfy (20), the inequality (50) hold and $\hat{H}\left(\vartheta_{p}\right)<0$. Then

$$
\hat{H}(\vartheta)<0, \vartheta \in\left[\vartheta_{p}, \vartheta_{0}\right] .
$$

The proof of the lemma is presented in Appendix A.

In this case, there is no singular region or capture zone. For all $\vartheta \in\left[\vartheta_{p}, \vartheta_{0}\right)$, the optimal strategies are $\hat{u}(\vartheta, w)=\hat{v}(\vartheta, w)=\operatorname{sign}(w), w \neq 0$, while the game value is given by 49.)

Remark 5. Due to the results of this section, in all cases either the game value $J_{\text {aux }}^{*}$ is zero or it is given by (49).

\section{SHRCP solution}

Due to 34 and $(38)$, the condition 35 becomes

$$
J_{\text {aux }}^{*}=z^{*}\left(0, \vartheta_{p}, \omega_{e}, \omega_{p 2}\right) .
$$

If for the initial position $\left(\vartheta_{0}, z_{0}\right), J_{\text {aux }}^{*}=0$, then the condition (52) is not satisfied. This means that the value $\left|z_{0}\right|$ can be increased such that the game value is given by (49), implying the inclusion $\left(\vartheta_{0}, z_{0}\right) \in \hat{D}_{1}$. By solving Eqn. (52) with respect to $\left|z_{0}\right|$,

$$
\begin{aligned}
\left|z_{0}\right|= & \mathcal{H}\left(\vartheta_{p}, \vartheta_{0}\right) \\
\triangleq & \int_{\vartheta_{p}}^{\vartheta_{0}} H\left(\xi, \omega_{e}, \omega_{p 1}\right) \mathrm{d} \xi+\int_{0}^{\vartheta_{p}} H\left(\xi, \omega_{e}, \omega_{p 2}\right) \mathrm{d} \xi \\
& +\delta a_{p 1}^{\max }\left(\exp \left(\left(\vartheta_{p}-\vartheta_{0}\right) / \tau_{p 1}\right)-1\right) .
\end{aligned}
$$

For any fixed $\vartheta_{0}$, the function $\mathcal{H}\left(\vartheta_{p}, \vartheta_{0}\right)$ is considered on the set $\Theta_{p}\left(\vartheta_{0}\right)=\left\{\vartheta_{p} \in\left(0, \vartheta_{0}\right): \mathcal{H}\left(\vartheta_{p}, \vartheta_{0}\right) \geq 0\right\}$.

Similarly to Shinar et al. (2009), we obtain that the derivative of $\mathcal{H}\left(\vartheta_{p}, \vartheta_{0}\right)$ with respect to $\vartheta_{p}$ has two zeros on the interval $\vartheta_{p} \in(-\infty,+\infty)$. One of these zeros is $\vartheta_{p}=0$, while the second is, subject to the condition

$$
\begin{gathered}
a_{p 2}^{\max }>a_{p 1}^{\max }, \\
\vartheta_{p}=\vartheta_{p}^{*} \triangleq \vartheta_{0}-\Delta \vartheta_{p},
\end{gathered}
$$

where

$$
\Delta \vartheta_{p}=\tau_{p 1} \ln \frac{a_{p 1}^{\max }\left(\tau_{p 2}-\tau_{p 1}\right)}{\tau_{p 1}\left(a_{p 2}^{\max }-a_{p 1}^{\max }\right)} .
$$

Like in the work of Shinar et al. (2009), it is shown that $\vartheta_{p}=\vartheta_{p}^{*}$ is the unique point of the local maximum of the function $\mathcal{H}\left(\vartheta_{p}, \vartheta_{0}\right)$ with respect to $\vartheta_{p} \in(-\infty,+\infty)$.

Note that the value of $\Delta \vartheta_{p}$ is independent of $\vartheta_{0}$. The obtained value of $\vartheta_{p}^{*}$ is feasible if $\vartheta_{p}^{*} \in \Theta_{p}\left(\vartheta_{0}\right)$. The latter implies two inequalities:

$$
\begin{gathered}
0<\Delta \vartheta_{p}<\vartheta_{0}, \\
\mathcal{H}\left(\vartheta_{p}^{*}, \vartheta_{0}\right) \geq 0 .
\end{gathered}
$$

If the condition (57) is valid, $\vartheta_{p}=\vartheta_{p}^{*}$ is the unique absolute maximum point of the function $\mathcal{H}\left(\vartheta_{p}, \vartheta_{0}\right)$ with respect to $\vartheta_{p} \in\left[0, \vartheta_{0}\right]$. Moreover, if both conditions (57)-(58) hold, then $\vartheta_{p}^{*}$ is the optimal switch point. If (57) holds and the point $\left(\vartheta_{0}, \mathcal{H}\left(\vartheta_{p}^{*}, \vartheta_{0}\right)\right)$ lies above the upper boundary of the union $C_{0}\left(\omega_{e}, \omega_{p 1}\right) \cup C_{0}\left(\omega_{e}, \omega_{p 2}\right)$, then it is a point of the upper boundary of $\mathcal{C}_{p}$. Note that this condition guarantees the fulfilment of (58).

If $0<\vartheta_{0} \leq \Delta \vartheta_{p}$, then the condition (57) is not satisfied. In this case, due to Shinar et al. (2009), the maximum of the function $\mathcal{H}\left(\vartheta_{p}, \vartheta_{0}\right)$ with respect to $\vartheta_{p} \in\left[0, \vartheta_{0}\right]$ is attained for $\vartheta_{p}^{*}=0$. This point is feasible if

$$
\begin{aligned}
& \mathcal{H}\left(0, \vartheta_{0}\right) \\
& =\int_{0}^{\vartheta_{0}} H\left(\xi, \omega_{e}, \omega_{p 1}\right) \mathrm{d} \xi \geq 0, \quad \forall \vartheta_{0} \in\left(0, \Delta \vartheta_{p}\right] .
\end{aligned}
$$

If the capture zone $C_{0}\left(\omega_{e}, \omega_{p 1}\right)$ is unbounded, the latter is satisfied for all $\Delta \vartheta_{p}>0$. Therefore, in this case, for $\vartheta_{0} \leq \Delta \vartheta_{p}$, the pursuer should employ its first dynamic mode on the entire interval $\left[0, \vartheta_{0}\right]$, and the boundary of $\mathcal{C}_{p}$ coincides with the boundary of $C_{0}\left(\omega_{e}, \omega_{p 1}\right)$.

If the capture zone $C_{0}\left(\omega_{e}, \omega_{p 1}\right)$ is bounded, the condition (59) is satisfied if and only if

$$
0<\Delta \vartheta_{p} \leq \vartheta_{c 1}
$$

Thus, in this case, for $\vartheta_{0} \leq \Delta \vartheta_{p}$, if the condition 60] holds, the boundary of $\mathcal{C}_{p}$ coincides with the boundary of $C_{0}\left(\omega_{e}, \omega_{p 1}\right)$.

For further analysis, we distinguish two cases: (A) $\left(\omega_{e}, \omega_{p 2}\right)$ satisfies (15), where the capture zone $C_{0}\left(\omega_{e}, \omega_{p 2}\right)$ is unbounded, and (B) $\left(\omega_{e}, \omega_{p 2}\right)$ satisfies (20), where the capture zone $C_{0}\left(\omega_{e}, \omega_{p 2}\right)$ is bounded. 
If $\left(\omega_{e}, \omega_{p}\right)$ satisfies 20, for further analysis, let us define the function

$$
Z^{*}\left(\vartheta, \omega_{e}, \omega_{p}\right) \triangleq \begin{cases}z^{*}\left(\vartheta, 0, \omega_{e}, \omega_{p}\right), & 0<\vartheta \leq \vartheta_{c}, \\ 0, & \vartheta_{c}<\vartheta \leq \vartheta_{0} .\end{cases}
$$

5.1. Construction of $\mathcal{C}_{p}$ in Case $\mathrm{A}$. Since the case where the capture zones for both pursuer dynamic modes are unbounded was studied by Shinar et al. (2009), we consider here case where the pair $\left(\omega_{e}, \omega_{p 1}\right)$ satisfies (20), i.e., the capture zone $C_{0}\left(\omega_{e}, \omega_{p 1}\right)$ is bounded. Due to (56), the inequality 57 is equivalent to the inequality

$$
1<\frac{a_{p 1}^{\max }\left(\tau_{p 2}-\tau_{p 1}\right)}{\tau_{p 1}\left(a_{p 2}^{\max }-a_{p 1}^{\max }\right)}<\exp \left(\vartheta_{0} / \tau_{p 1}\right) .
$$

Lemma 2. Let the condition (62) be valid. Then, the point $\left(\vartheta_{0}, \mathcal{H}\left(\vartheta_{p}^{*}, \vartheta_{0}\right)\right)$ lies above the upper boundary of the union $C_{0}\left(\omega_{e}, \omega_{p 1}\right) \cup C_{0}\left(\omega_{e}, \omega_{p 2}\right)$.

The proof of the lemma is presented in Appendix B.

Remember that $\Delta \vartheta_{p}$ is independent of $\vartheta_{0}$. Then, based on Lemma 2 and the inequalities (59)- (60), we obtain, by replacing $\vartheta_{0}$ with any $\vartheta \in\left(0, \vartheta_{0}\right]$, the following theorem.

Theorem 1. In Case A, the SHRCP is solvable if the conditions (62) and (60) hold. The capture zone of the pursuer's hybrid dynamics is

$$
\mathcal{C}_{p}=\left\{(\vartheta, z): \vartheta \in\left[0, \vartheta_{0}\right],|z| \leq \mathcal{Z}_{p}(\vartheta)\right\}
$$

where

$$
\mathcal{Z}_{p}(\vartheta)=\left\{\begin{array}{cc}
\mathcal{H}\left(\vartheta-\Delta \vartheta_{p}, \vartheta\right), & \Delta \vartheta_{p}<\vartheta \leq \vartheta_{0}, \\
z^{*}\left(\vartheta, 0, \omega_{e}, \omega_{p 1}\right), & 0<\vartheta \leq \Delta \vartheta_{p} .
\end{array}\right.
$$

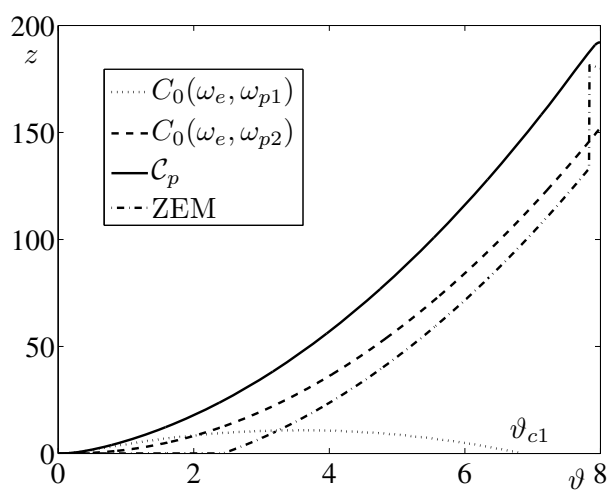

Fig. 13. Hybrid capture zone in Case A.

In Fig. 13, the upper boundaries of $C_{0}\left(\omega_{e}, \omega_{p 1}\right)$, $C_{0}\left(\omega_{e}, \omega_{p 2}\right), \mathcal{C}_{p}$ and the ZEM trajectory are depicted for the data of Table 3 and for $\vartheta_{0}=8 \mathrm{~s}$. For these data, $C_{0}\left(\omega_{e}, \omega_{p 1}\right)$ is bounded $\left(\vartheta_{c 1}=6.85 \mathrm{~s}\right)$ while $C_{0}\left(\omega_{e}, \omega_{p 2}\right)$ is unbounded. In this example, $\Delta \vartheta_{p}=0.17 \mathrm{~s}$ $\left(\Delta \vartheta_{p}<\vartheta_{c 1}\right)$, i.e., the condition (58) is satisfied. The ZEM trajectory emanates from the initial point $\left(\vartheta_{0}, z_{0}\right)=$ $(8,180): \quad\left(\vartheta_{0}, z_{0}\right) \notin C_{0}\left(\omega_{e}, \omega_{p 1}\right) \cup C_{0}\left(\omega_{e}, \omega_{p 2}\right)$ and $\left(\vartheta_{0}, z_{0}\right) \in \mathcal{C}_{p}$. This trajectory is generated by the strategies

$$
u=v= \begin{cases}\operatorname{sign}(w), & \vartheta_{0}-\Delta \vartheta_{p}<\vartheta \leq \vartheta_{0}, \\ \operatorname{sign}(z), & 0 \leq \vartheta<\vartheta_{0}-\Delta \vartheta_{p}\end{cases}
$$

It is seen that, for $\vartheta \in[0,8], \mathcal{C}_{p} \supset C_{0}\left(\omega_{e}, \omega_{p 1}\right) \cup$ $C_{0}\left(\omega_{e}, \omega_{p 2}\right)$, and the ZEM trajectory terminates at the point $(0,0)$.

5.2. Construction of $\mathcal{C}_{p}$ in Case $B$. If, in this case, the pair $\left(\omega_{e}, \omega_{p 1}\right)$ satisfies [15), then $a_{p 1}^{\max }>a_{e}^{\max }, a_{p 2}^{\max }<$ $a_{e}^{\max }$, leading to $a_{p 2}^{\max }<a_{p 1}^{\max }$, i.e., the condition (54) is not satisfied. In this case, $\partial \mathcal{H}\left(\vartheta_{p}, \vartheta_{0}\right) / \partial \vartheta_{p} \leq 0$, for all $\vartheta_{p} \in(-\infty,+\infty)$, i.e., the maximal value of $\mathcal{H}\left(\vartheta_{p}, \vartheta_{0}\right)$ with respect to $\vartheta_{p} \in\left[0, \vartheta_{0}\right]$ is attained for $\vartheta_{p}=0$. The latter means that the pursuer should use its first dynamic mode on the entire interval $\left[0, \vartheta_{0}\right]$, which does not lead to increasing its capturability.

Thus, in Case B, the only possibility to increase the pursuer's capturability is when the pair $\left(\omega_{e}, \omega_{p 1}\right)$ satisfies [20), i.e., both capture zones $C_{0}\left(\omega_{e}, \omega_{p 1}\right)$ and $C_{0}\left(\omega_{e}, \omega_{p 2}\right)$ are bounded, meaning that they exist for $\vartheta \in\left[0, \vartheta_{c 1}\right]$ and $\vartheta \in\left[0, \vartheta_{c 2}\right]$, respectively.

Remark 6. If $\vartheta_{c 2}<\vartheta_{p}<\vartheta_{0}$, then $z^{*}\left(0, \vartheta_{p}, \omega_{e}, \omega_{p 2}\right)<$ 0 , meaning that the equality (52) is impossible. Therefore, in the case $\mathrm{B}$, the maximum of $\mathcal{H}\left(\vartheta_{p}, \vartheta_{0}\right)$ should be searched for $\vartheta_{p} \in\left[0, \vartheta_{c 2}\right]$. If $\vartheta_{p}^{*} \in\left(0, \vartheta_{c 2}\right]$ and the condition (58) holds, $\vartheta_{p}^{*}$ is the optimal switch moment. If $\vartheta_{p}^{*}>\vartheta_{c 2}$, then the maximum value of $\mathcal{H}\left(\vartheta_{p}, \vartheta_{0}\right)$ is attained for $\vartheta_{p}=\vartheta_{c 2}$, which becomes the optimal switch moment subject to the condition

$$
\mathcal{H}\left(\vartheta_{c 2}, \vartheta_{0}\right)=\int_{\vartheta_{c 2}}^{\vartheta_{0}} H\left(\xi, \omega_{e}, \omega_{p 1}\right) \mathrm{d} \xi+\Delta Z\left(\vartheta_{c 2}\right) \geq 0 .
$$

Remark 7. Note that the inclusion $\vartheta_{p}^{*} \in\left(0, \vartheta_{c 2}\right]$ is equivalent to the inequality

$$
\exp \left(\frac{\vartheta_{0}-\vartheta_{c 2}}{\tau_{p 1}}\right) \leq \frac{a_{p 1}^{\max }\left(\tau_{p 2}-\tau_{p 1}\right)}{\tau_{p 1}\left(a_{p 2}^{\max }-a_{p 1}^{\max }\right)}<\exp \left(\frac{\vartheta_{0}}{\tau_{p 1}}\right) .
$$

Based on Remarks 6 and 7 similarly to Theorem 1 we have the following statement.

Theorem 2. Let the pairs $\left(\omega_{e}, \omega_{p i}\right)$ satisfy (20), $i=$ 1,2 . Then two following SHRCP solvability conditions are valid: 
I. When the inequality (66) holds, then the SHRCP is solvable if for $\vartheta \in\left(\Delta \vartheta_{p}, \vartheta_{0}\right]$,

$\mathcal{H}\left(\vartheta-\Delta \vartheta_{p}, \vartheta\right)>\max \left\{Z^{*}\left(\vartheta, \omega_{e}, \omega_{p 1}\right), Z^{*}\left(\vartheta, \omega_{e}, \omega_{p 2}\right)\right\}$.

In this case, $\mathcal{C}_{p}$ is given by (63)-(64).

II. When the inequality (66) is not satisfied, then the SHRCP is solvable if, for $\vartheta \in\left(\Delta \vartheta_{p}, \vartheta_{c 2}+\Delta \vartheta_{p}\right]$, [67) is satisfied and, for $\vartheta \in\left(\vartheta_{c 2}+\Delta \vartheta_{p}, \vartheta_{0}\right]$,

$$
\mathcal{H}\left(\vartheta_{c 2}, \vartheta\right)>Z^{*}\left(\vartheta, \omega_{e}, \omega_{p 1}\right) .
$$

In this case, $\mathcal{C}_{p}$ is given by (63), where

$$
\mathcal{Z}_{p}(\vartheta)=\left\{\begin{array}{lc}
\mathcal{H}\left(\vartheta_{c 2}, \vartheta\right), & \vartheta_{c 2}+\Delta \vartheta_{p}<\vartheta \leq \vartheta_{0}, \\
\mathcal{H}\left(\vartheta-\Delta \vartheta_{p}, \vartheta\right), & \Delta \vartheta_{p}<\vartheta \leq \vartheta_{c 2}+\Delta \vartheta_{p}, \\
z^{*}\left(\vartheta, 0, \omega_{e}, \omega_{p 1}\right), & 0<\vartheta \leq \Delta \vartheta_{p} .
\end{array}\right.
$$

Remark 8. Similarly to Lemma2, it is shown that, if

$$
\vartheta_{c 1} \leq \vartheta_{c 2}
$$

and

$$
0<\Delta \vartheta_{p} \leq \vartheta_{c 2},
$$

then for all $\vartheta \in\left(\Delta \vartheta_{p}, \vartheta_{c 2}\right]$ the inequality 67 is valid.

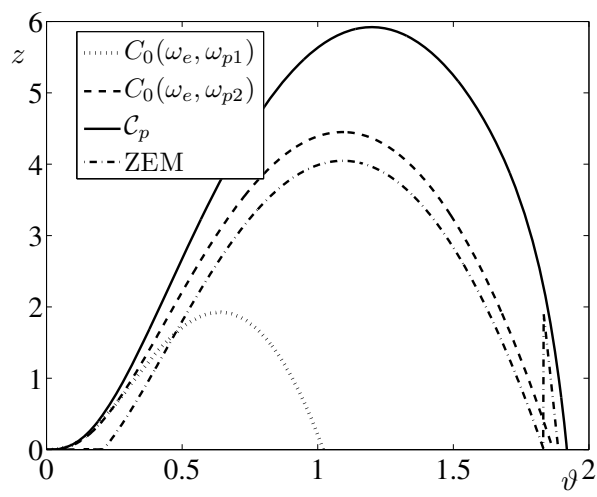

Fig. 14. Hybrid capture zone in Case B.

In Fig. 14 the upper boundaries of $C_{0}\left(\omega_{e}, \omega_{p 1}\right)$, $C_{0}\left(\omega_{e}, \omega_{p 2}\right), \mathcal{C}_{p}$ and the ZEM trajectory are depicted for the data of Table 2 and for $\vartheta_{0}=1.92 \mathrm{~s}$. For these data, both $C_{0}\left(\omega_{e}, \omega_{p 1}\right)$ and $C_{0}\left(\omega_{e}, \omega_{p 2}\right)$ are bounded $\left(\vartheta_{c 1}=1.02 \mathrm{~s}, \vartheta_{c 2}=1.87 \mathrm{~s}\right)$. In this example, $\Delta \vartheta_{p}=0.056 \mathrm{~s}$, leading to $\vartheta_{p}^{*}=1.86 \mathrm{~s}<\vartheta_{c 2}$. The condition (66) is satisfied. The ZEM trajectory emanates from the initial point $\left(\vartheta_{0}, z_{0}\right)=(1.9,0):\left(\vartheta_{0}, z_{0}\right) \notin$ $C_{0}\left(\omega_{e}, \omega_{p 1}\right) \cup C_{0}\left(\omega_{e}, \omega_{p 2}\right)$ and $\left(\vartheta_{0}, z_{0}\right) \in \mathcal{C}_{p}$. This trajectory is generated by the strategies (65). It is seen that, for $\vartheta \in[0,1.92], \mathcal{C}_{p} \supset C_{0}\left(\omega_{e}, \omega_{p 1}\right) \cup C_{0}\left(\omega_{e}, \omega_{p 2}\right)$, and the ZEM trajectory terminates at the point $(0,0)$.
5.3. Pursuer's robust capturing strategy. Based on the results of Sections 2.3 and 4, the pursuer's strategy, guaranteeing the capture from any initial position $\left(\vartheta_{0}, z_{0}\right) \in \mathcal{C}_{p}$, robustly with respect to any admissible evader behavior, is the following. If $\left(\vartheta_{0}, z_{0}\right)$ belongs to at least one of the capture zones $\operatorname{clo}\left(C_{0}\left(\omega_{e}, \omega_{p i}\right)\right), i=$ 1,2 , then the pursuer chooses the corresponding dynamic mode and the corresponding optimal strategy, and does not switch from this dynamic mode to the other till the end of the interception. If $\left(\vartheta_{0}, z_{0}\right) \notin C_{0}\left(\omega_{e}, \omega_{p 1}\right) \cup$ $C_{0}\left(\omega_{e}, \omega_{p 2}\right)$, then at the beginning the pursuer chooses the first dynamic mode $\omega_{p 1}$. Also, it chooses the optimal switch moment $\vartheta_{p}=\bar{\vartheta}_{p}^{*}$ from the dynamic mode $\omega_{p 1}$ to the dynamic mode $\omega_{p 2}$. Due to results of two previous subsections, $\bar{\vartheta}_{p}^{*}$ can be either $\vartheta_{p}^{*}=\vartheta_{0}-\Delta \vartheta_{p}$ or $\vartheta_{c 2}$. Then, on the interval $\vartheta \in\left(\bar{\vartheta}_{p}^{*}, \vartheta_{0}\right]$ (before the switch) the pursuer uses the strategy $u\left(\vartheta, z, a_{p}\right)=\operatorname{sign}(w)$, while on the interval $\vartheta \in\left[0, \bar{\vartheta}_{p}^{*}\right)$ (after the switch) the pursuer uses the strategy $u(\vartheta, z)=\operatorname{sign}(z)$.

Remark 9. The robustness of the capture zones $\mathcal{C}_{p}$ constructed in Theorems 1 and 2 and the robustness of the pursuer's capturing strategy mean the following. If the pursuit starts in $\mathcal{C}_{p}$, then by using the capturing strategy the pursuer guarantees the capture (zero miss distance) at $t=t_{f}(\vartheta=0)$ against arbitrary admissible evader control. Indeed, at the first stage, including the switch moment, the pursuer uses its optimal strategy in the ADGD, thus steering the trajectory into the capture zone, corresponding to its second dynamic mode. At the second stage, after the switch, the pursuer employs its optimal strategy in the SFDG with the second dynamic mode, steering the trajectory to zero. Since the pursuer strategies at both stages are the optimal differential game strategies, they take into account the worst case evader admissible control, and therefore are definitely robust with respect to the evader's behavior.

Remark 10. The approach developed in this paper can be extended to analysis of the reliability issue. Indeed, the abrupt pursuer actuator fault, i.e., deterioration either of the maneuverability (decreasing $a_{p}^{\max }$ ) or of the agility (decreasing the value $a_{p}^{\max } / \tau_{p}$ ), can be considered in the framework of the hybrid dynamics. In this case, by using a method similar to the one described in this paper, the pursuer's robust and reliable capture zone can be constructed.

\section{Simulation results}

In order to evaluate the theoretical results, numerical simulations were carried out under the following realistic assumptions: (I) nonlinear engagement model, (II) random wind disturbance. Due to these assumptions, the 
engagement is described by

$$
\begin{aligned}
\dot{x}_{e} & =V_{e} \cos \varphi_{e}+W_{x e}(t), & & x_{e}(0)=r_{0}, \\
\dot{y}_{e} & =V_{e} \sin \varphi_{e}+W_{y e}(t), & & y_{e}(0)=0, \\
\dot{\varphi}_{e} & =a_{e} / V_{e}, & \varphi_{e}(0) & =\varphi_{e 0}, \\
\dot{a}_{e} & =\left(a_{e}^{\max } v-a_{e}\right) / \tau_{e}, & & a_{e}(0)=0, \\
\dot{x}_{p} & =V_{p} \cos \varphi_{p}+W_{x p}(t), & & x_{p}(0)=0, \\
\dot{y}_{p} & =V_{p} \sin \varphi_{p}+W_{y p}(t), & y_{p}(0) & =0, \\
\dot{\varphi}_{p} & =a_{p} / V_{p}, & \varphi_{p}(0) & =\varphi_{p 0}, \\
\dot{a}_{p} & =\left(a_{p}^{\max } u-a_{p}\right) / \tau_{p}, & a_{p}(0) & =0,
\end{aligned}
$$

where $W_{i}(t)=\left[W_{x i}(t), W_{y i}(t)\right]^{T}, i=e, p$, are time-varying wind velocity vectors, influencing the evader and the pursuer, respectively.

In the wind model, it is assumed that the magnitude and the direction angle of the wind are combined from constant and time-varying parts. The constant part is the wind magnitude $A_{0}$ and direction angle $\psi_{0}$ at the vehicles' collision point. The time-varying part is considerable when the vehicles are far from the collision point, while it decreases when the vehicles approach this point and vanishes at this point. Let $A_{i 0}$ and $\psi_{i 0}, i=e, p$, be the magnitude and the angle of the wind, influencing the evader and the pursuer, respectively, for $t=0$. Then, by assuming additionally the linear time dependance of the magnitudes and the direction angles of the wind, influencing the vehicles, we can represent these functions as

$$
\begin{aligned}
& A_{i}(t)=A_{0}+\left(1-t / t_{f}\right) \Delta A_{i}, \\
& \psi_{i}(t)=\psi_{0}+\left(1-t / t_{f}\right) \Delta \psi_{i},
\end{aligned}
$$

$i=e, p$, where $\Delta A_{i} \triangleq A_{i 0}-A_{0}, \Delta \psi_{i} \triangleq \psi_{i 0}-\psi_{0}, i=$ $e, p$. Thus, the components of the wind velocity vectors in the $x$ - and $y$-directions are

$$
\begin{aligned}
& W_{x i}(t)=A_{i}(t) \cos \psi_{i}(t), \\
& W_{y i}(t)=A_{i}(t) \sin \psi_{i}(t),
\end{aligned}
$$

$i=e, p$, and $t \in\left[0, t_{f}\right]$.

In this simulation study, the magnitude and the direction of the wind are modeled following Wolf et al. (2010). Namely, the values $A_{0}, \Delta A_{i}, i=e, p$, were chosen as normally distributed random ones: $A_{0} \sim$ $N\left(\mu_{A_{0}}, \sigma_{A_{0}}\right), \Delta A_{i} \sim N\left(\mu_{\Delta A_{i}}, \sigma_{\Delta A_{i}}\right), i=e, p$. For the values $\psi_{0}, \Delta \psi_{i}, i=e, p$, the von Mises distribution (Jammalamadaka and Sengupta, 2001) was employed. It is an analogue of a Gaussian distribution on the circle. Its probability density function is

$$
f_{\mathrm{VM}}(\psi ; \mu, \kappa)=\frac{\exp (\kappa(\mu-\psi))}{2 \pi I_{0}(\kappa)},
$$

where $I_{0}(\kappa)$ is the modified Bessel function of the first kind of order $0, \mu$ is the mean, $\kappa$ characterizes the concentration of the random value around $\mu$. In what follows, we write the random value, distributed in accordance with (77), as $\psi \sim V M(\mu, \kappa)$, and assume $\psi_{0} \sim V M\left(\mu_{\psi_{0}}, \kappa_{\psi_{0}}\right), \Delta \psi_{i} \sim V M\left(\mu_{\Delta \psi_{i}}, \kappa_{\Delta \psi_{i}}\right)$, $i=e, p$.

In the simulation, the pursuer uses the optimal strategy, described in Section 5.3 For the evader, two different control types were employed:

(a) the bang-bang control with a single fixed switch moment $t_{s w} \in\left(0, t_{f}\right)$ :

$$
v= \begin{cases}1, & 0 \leq t<t_{s w}, \\ -1, & t_{s w} \leq t<t_{f},\end{cases}
$$

(b) the bang-bang control (78) with a random switch moment $t_{s w} \sim U\left[0, t_{f}\right]$.

Remark 11. The evader controls (a) and (b) are widely used in the literature (Shinar et al., 2007; Glizer and Turetsky, 2009; Shinar and Turetsky, 2009; 2013a; 2013b)

In the simulation, the vehicles' dynamics data are chosen from Table 3. The vehicles' velocities are $V_{e}=$ $2700 \mathrm{~m} / \mathrm{s}$ and $V_{p}=2300 \mathrm{~m} / \mathrm{s}$, the initial range between the vehicles is $r_{0}=40 \mathrm{~km}$, the initial values of the vehicles aspect angles are $\varphi_{e 0}=2.2 \mathrm{deg}, \varphi_{p 0}=2 \mathrm{deg}$, resulting in $t_{f}=\vartheta_{0}=8 \mathrm{~s}, z_{0}=187.1 \mathrm{~m}$. Note that (see Fig. 13)

$$
\left(\vartheta_{0}, z_{0}\right) \notin C_{0}\left(\omega_{e}, \omega_{p 1}\right) \cup C_{0}\left(\omega_{e}, \omega_{p 2}\right), \quad\left(\vartheta_{0}, z_{0}\right) \in \mathcal{C}_{p}
$$

The wind model data is chosen from Table 4

Table 4. Wind amplitude and angle simulation data.

\begin{tabular}{|c|c|c|c|}
\hline & $A_{0}[\mathrm{~m} / \mathrm{s}]$ & $\Delta A_{e}[\mathrm{~m} / \mathrm{s}]$ & $\Delta A_{p}[\mathrm{~m} / \mathrm{s}]$ \\
\hline \hline$\mu$ & 10 & 15 & 12 \\
\hline$\sigma$ & 2 & 3 & 2.4 \\
\hline & $\psi_{0}[\mathrm{rad}]$ & $\Delta \psi_{e}[\mathrm{rad}]$ & $\Delta \psi_{p}[\mathrm{rad}]$ \\
\hline$\mu$ & $0: \pi / 4: \pi$ & 0 & 0 \\
\hline$\kappa$ & 100 & 0.15 & 0.2 \\
\hline
\end{tabular}

Remark 12. In the simulation, the miss distance cannot be calculated as $\left|y_{e}\left(t_{f}\right)-y_{p}\left(t_{f}\right)\right|$ and it is defined as

$$
\tilde{r}_{f}=\min _{t \geq 0} \sqrt{\left(x_{e}(t)-x_{p}(t)\right)^{2}+\left(y_{e}(t)-y_{p}(t)\right)^{2}} .
$$

In Fig.15, the average miss distance for various mean values $\mu_{\psi_{0}}$ of the wind direction angle at the vehicles' collision point is depicted for the evader control (78) as a function of $t_{s w}$. The pursuer employs its robust capturing hybrid strategy, described in Section 5.3. The average miss distance is obtained by 100 Monte Carlo simulations 
for each switch moment $t_{s w}=0: 0.08: 8$. It is seen that for each $\mu_{\psi_{0}}$ the presence of the wind increases the average miss distance when $t_{s w}$ approaches $t_{f}$. Moreover, the worst case $\mu_{\psi_{0}}$ is $90 \mathrm{deg}$. Nevertheless, the maximal average miss distance is less than $90 \mathrm{~cm}$.

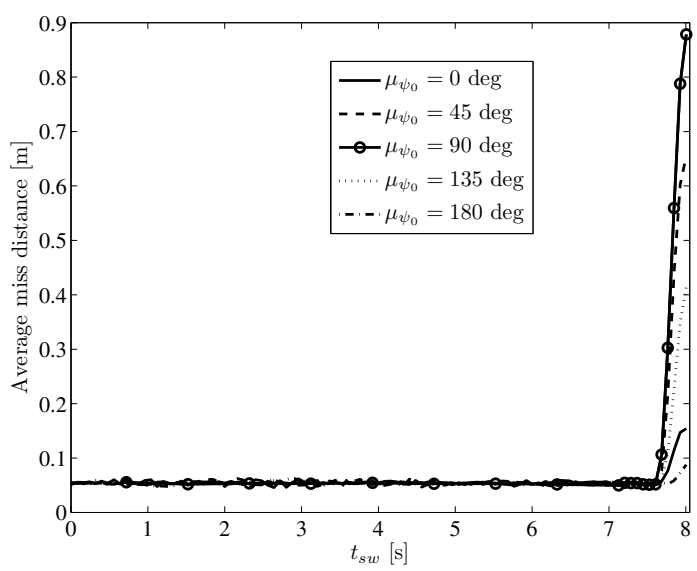

Fig. 15. Average miss distance.

In Figs. 16 and 17 the cumulative distribution of the miss distance is shown for the pursuer's robust capturing hybrid strategy (Fig. 16) and fixed dynamics optimal strategy (Fig. 17) against the evader's bang-bang strategy with random switch. In these simulations, $\mu_{\psi_{0}}=90 \mathrm{deg}$. The number of Monte Carlo runs was 500. It is seen that the robust capturing hybrid strategy improves considerably the outcome of the interception, in comparison with the fixed dynamics optimal strategy. Namely, the huge 95th miss distance percentiles $197.3 \mathrm{~m}$ and $35.4 \mathrm{~m}$, obtained by using the optimal strategy for the first and the second dynamics modes, are reduced to $9.2 \mathrm{~cm}$, obtained by using the robust capturing hybrid strategy. This result is a direct consequence of Eqn. (79). The simulation with different mean values of the wind direction angle at the vehicles' collision point showed that the value of $\mu_{\psi_{0}}$ does not influence significantly on the miss distance cumulative distribution, including its 95 th percentile.

\section{Conclusions}

In this paper, a robust interception problem of a maneuverable fixed dynamics target by a hybrid dynamics interceptor with two different dynamic modes was considered. The solution of this problem utilizes the discontinuity of the zero-effort miss distance.

Previously, it was shown that the interceptor's capturability can be increased by using hybrid dynamics in the case where for both dynamic modes the interceptor is superior in maneuverability and agility. In the present paper, two additional cases of capturability increasing are established: (A) for the first dynamic

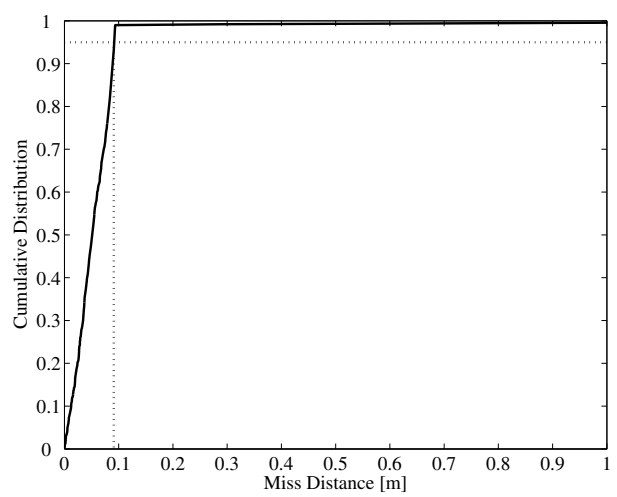

Fig. 16. Pursuer's robust capturing hybrid strategy against the evader's bang-bang strategy with random switch.

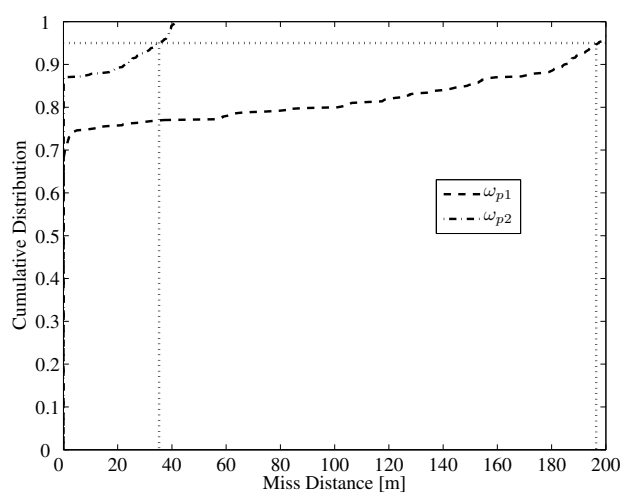

Fig. 17. Pursuer's fixed dynamics optimal strategy against the evader's bang-bang strategy with random switch.

mode, the interceptor is inferior in maneuverability and superior in agility; for the second, it is superior both in maneuverability and agility, (B) for both dynamic modes, the interceptor is inferior in maneuverability and superior in agility. In both the cases, the respective capture zones are constructed. The pursuer's robust capturing strategy is derived.

The theoretical results were evaluated by extensive simulation for the realistic non-linear engagement model, in the presence of a random wind and various types of evader control. The simulation results show that: (a) the pursuer's robust capture zone and robust capturing strategy, constructed based on the linearized engagement model and the hybrid dynamics of the pursuer, are also valid for the non-linear engagement model, (b) if the engagement starts in the pursuer's robust capture zone, its robust capturing strategy is low-sensitive to the random wind, (c) employing the hybrid dynamics of the pursuer improves dramatically the interception outcome, comparing with the fixed dynamics cases.

The future issues of the topic, requiring further investigations, are (i) construction of the robust escape zone of the hybrid dynamics evader in the cases where at 
least for one of its dynamic modes the pursuer's capture zone is bounded, (ii) a study of capture and escape zones for both the pursuer and the evader having hybrid dynamics, (iii) a study of the reliability of the pursuer's robust capturing strategy and the evader's robust escaping strategy when at least one of the counterparts has hybrid dynamics.

\section{References}

Bartolini, G. and Zolezzi, T. (1986). Control of nonlinear variable structure systems, Journal of Mathematical Analysis and Applications 118(1): 42-62.

Chen, X. and Fukuda, T. (1997). Variable structure system theory based disturbance identification and its applications, International Journal of Control 68(2): 373-384.

Gao, Y., Lygeros, J. and Quincampoix, M. (2007). On the reachability problem for uncertain hybrid systems, IEEE Transactions on Automatic Control 52(9): 1572-1586.

Glizer, V.Y. (1996). Optimal planar interception with fixed end conditions: A closed form solution, Journal of Optimization Theory and Applications 88(3): 503-539.

Glizer, V.Y. (1997). Optimal planar interception with fixed end conditions: Approximate solutions, Journal of Optimization Theory and Applications 93(1): 1-25.

Glizer, V.Y. (1999). Homicidal chauffeur game with target set in the shape of a circular angular sector: Conditions for existence of a closed barrier, Journal of Optimization Theory and Applications 101(3): 581-598.

Glizer, V.Y. and Turetsky, V. (2008). Complete solution of a differential game with linear dynamics and bounded controls, Applied Mathematics Research Express, Article ID: abm012.

Glizer, V.Y. and Turetsky, V. (2009). A linear differential game with bounded controls and two information delays, Optimal Control, Applications and Methods 30(2): 135-161.

Glizer, V.Y. and Turetsky, V. (2012). Robust Controllability of Linear Systems, Nova Science Publishers Inc., New York, NY.

Glizer, V.Y., Turetsky, V., Fridman, L. and Shinar, J. (2012). History-dependent modified sliding mode interception strategies with maximal capture zone, Journal of the Franklin Institute 349(2): 638-657.

Gutman, S. (2006). Applied Min-Max Approach to Missile Guidance and Control, Progress in Astronautics and Aeronautics, Vol. 209, AIAA, Inc., Reston, VI.

Gutman, S. and Leitmann, G. (1976). Optimal strategies in the neighborhood of a collision course, AIAA Journal 14(9): 1210-1212.

Ho, Y.C., Bryson, A.E. and Baron, S. (1965). Differential games and optimal pursuit-evasion strategies, IEEE Transactions on Automatic Control 10(4): 385-389.

Isaacs, R. (1965). Differential Games, John Wiley, New York, NY.
Jammalamadaka, S.R. and Sengupta, A. (2001). Topics in Circular Statistics, World Scientific Publishing Company, Singapore.

Leitmann, G. (1980). Guaranteed avoidance strategies, Journal of Optimization Theory and Applications 32(4): 569-576.

Lin, H. and Antsaklis, P.J. (2003). Robust regulation of polytopic uncertain linear hybrid systems with networked control system applications, in P.J. Antsaklis and D. Liu (Eds.), Stability and Control of Dynamical Systems with Applications, Birkhauser, Boston, MA, Chapter 4, pp. 83-108.

Lygeros, J., Tomlin, C. and Sastry, S. (1999). Controllers for reachability specifications for hybrid systems, Automatica 35(3): 349-370.

Mitchell, I.M., Bayen, A.M. and Tomlin, C.J. (2005). A time-dependent Hamilton-Jacobi formulation of reachable sets for continuous dynamic games, IEEE Transactions on Automatic Control 50(7): 947-957.

Noura, H., Theilliol, D., Ponsart, J.-C. and Chamseddine, A. (2009). Fault-tolerant Control Systems. Design and Practical Application, Advances in Industrial Control, Springer, London.

Patsko, V.S. and Turova, V.L. (2004). Families of semipermeable curves in differential games with the homicidal Chauffeur dynamics, Automatica 40(12): 2059-2068

Poveda, J., Ochoa-Llerasy, N. and Rodriguez, C. (2012) Guidance of an autonomous glider based on proportional navigation and virtual targets: A hybrid dynamical systems approach, Proceedings of the AIAA Guidance, Navigation, and Control Conference 2012, Minneapolis, MN, USA pp. 1-21, Paper ID: AIAA 2012-4905.

Shima, T. and Shinar, J. (2002). Time-varying linear pursuit-evasion game models with bounded controls, Journal of Guidance, Control and Dynamics 25(3): 425-432.

Shinar, J. (1981). Solution techniques for realistic pursuit-evasion games, in C. Leondes (Ed.), Advances in Control and Dynamic Systems, Control and Dynamic Systems, Vol. 17, Academic Press, New York, NY pp. 63-124

Shinar, J., Glizer, V.Y. and Turetsky, V. (2009). A pursuit-evasion game with hybrid pursuer dynamics, $E u$ ropean Journal of Control 15(6): 665-684

Shinar, J., Glizer, V.Y. and Turetsky, V. (2010). Robust pursuit of a hybrid evader, Applied Mathematics and Computation 217(3): 1231-1245.

Shinar, J., Glizer, V.Y. and Turetsky, V. (2012). Complete solution of a pursuit-evasion differential game with hybric evader dynamics, International Game Theory Review 14(3): 1250014-1 - 1250014-31.

Shinar, J., Glizer, V.Y. and Turetsky, V. (2013). The effect of pursuer dynamics on the value of linear pursuit-evasion games with bounded controls, in V. Krivan and G. Zaccour (Eds.), Advances in Dynamic Games-Theory, Applications, and Numerical Methods, Annals of the International Society of Dynamic Games, Vol. 13, Birkhauser, Basel, pp. 313-350 
Shinar, J., Glizer, V.Y. and Turetsky, V. (2014). Capture zone of linear strategies in the interception problems with variable structure dynamics, Journal of the Franklin Institute 351(4): 2378-2395.

Shinar, J. and Turetsky, V. (2009). Three-dimensional validation of an integrated estimation/guidance algorithm against randomly maneuvering targets, Journal of Guidance, Control and Dynamics 32(3): 1034-1039.

Shinar, J. and Turetsky, V. (2013a). The estimator is the key element in interception endgames, Proceedings of the 5th European Conference for Aero-Space Sciences, Munich, Germany, pp. 1-3.

Shinar, J. and Turetsky, V. (2013b). On the crucial role of the estimation in interception endgames, Proceedings of EuroGNC 2013, 2nd CEAS Specialist Conference on Guidance, Navigation and Control, Delft, The Netherlands, pp. 499-506.

Shinar, J., Turetsky, V. and Oshman, Y. (2007). Integrated estimation/guidance design approach for improved homing against randomly maneuvering targets, Journal of Guidance, Control and Dynamics 30(1): 154-160.

Turetsky, V. and Shinar, J. (2003). Missile guidance laws based on pursuit-evasion game formulations, Automatica 39(4): 607-618.

Utkin, V.I. (1983). Variable structure systems: Present and future, Automation and Remote Control 44(9): 1105-1120.

Wolf, M.T., Blackmore, L., Kuwata, Y., Fathpour, N., Elfes, A. and Newman, C. (2010). Probabilistic motion planning of balloons in strong, uncertain wind fields, Proceedings of the 2010 IEEE International Conference on Robotics and Automation, Anchorage, AK, USA, pp. 1123-1129.

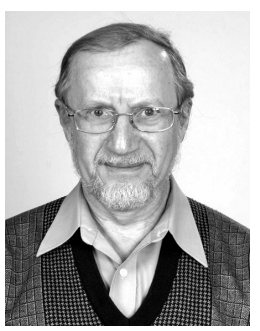

Valery Y. Glizer was born in Dniepropetrovsk, Ukraine, in 1950. He received the M.Sc. degree in mathematics from Dniepropetrovsk State University in 1972, and his Ph.D. degree in mathematics and physics from Moscow State University, Russia, in 1980. From 1990 to 2007, Prof. Glizer served as a senior research fellow at the Technion Israel Institute of Technology, Haifa, Israel. In 2007, he joined the Ort Braude College of Engineering, Karmiel, Israel. Currently, he is an associate professor at the Department of Applied Mathematics in this college. Prof. Glizer is an author/coauthor of more than 180 scientific publications, including the monograph Robust Controllability of Linear Systems (2012). He is an associate editor of a number of international journals. He has served as the chairman of IPCs for several international conferences and has been a member of IPCs of more than 20 international conferences. Prof. Glizer was awarded for the best paper at the 2011 International Conference on Applied and Engineering Mathematics of the World Congress of Engineering (WCE 2011), London, UK. His main research interests are dynamic games, optimal control, robust control, singular perturbations, and time delay systems.

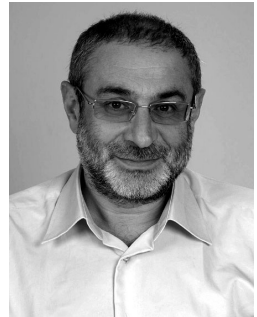

Vladimir Turetsky was born in 1959 in Ekaterinburg, Russia. In 1981, he received his M.Sc. in applied mathematics from Ural State University, Ekaterinburg. In 1988, he received his Ph.D. in applied mathematics from the Institute of Mathematics and Mechanics, Russian Academy of Sciences, Ekaterinburg. From 1981 to 1992, he was a research scientist at the Institute of Metrology, Ekaterinburg. From 1992 to 1999 he was with the Faculty of Mathematics and Mechanics, Ural State University, as an assistant professor and an associate professor. From 2000 to 2010, he was with the Faculty of Aerospace Engineering, Technion, as a senior research fellow. In 2010, Prof. Turetsky joined the Ort Braude College of Engineering, Karmiel, Israel. Currently, he is an associate professor at the Department of Applied Mathematics in this college. He is an author/coauthor of more than 120 scientific publications, including the monograph Robust Controllability of Linear Systems (2012). He has been a member of IPCs of several international conferences. Prof. Turetsky was awarded for the best paper at the $2011 \mathrm{In}-$ ternational Conference on Applied and Engineering Mathematics of the World Congress of Engineering (WCE 2011), London, UK. His main research interests are robust control, differential games and their applications in missile guidance.

\section{Appendix A}

\section{Proof of Lemma 1}

Since $\hat{H}\left(\vartheta_{p}\right)<0$, then, in order to prove (51), it is sufficient to show that

$$
\begin{aligned}
\frac{\mathrm{d} \hat{H}}{d \vartheta}= & \frac{\mathrm{d} H\left(\vartheta, \omega_{e}, \omega_{p 1}\right)}{\mathrm{d} \vartheta} \\
& +\frac{\delta a_{p 1}^{\max }}{\tau_{p 1}^{2}} \exp \left(\frac{\vartheta_{p}-\vartheta}{\tau_{p 1}}\right) \leq 0 .
\end{aligned}
$$

Due to 30 , the second term in A1 is negative. Hence, in order to prove the inequality in (A1), it is sufficient to prove that, for $\vartheta \in\left[\vartheta_{p}, \vartheta_{0}\right]$,

$$
\begin{aligned}
& \frac{\mathrm{d} H\left(\vartheta, \omega_{e}, \omega_{p 1}\right)}{\mathrm{d} \vartheta} \\
& =a_{p 1}^{\max }\left(1-\exp \left(-\frac{\vartheta}{\tau_{p 1}}\right)\right) \\
& \quad-a_{e}^{\max }\left(1-\exp \left(-\frac{\vartheta}{\tau_{e}}\right)\right) \\
& \leq 0 .
\end{aligned}
$$

Note that, due to the SFDG solution (Shinar, 1981; Shima and Shinar, 2002), there exists $\vartheta_{1} \in\left(0, \vartheta_{c 1}\right)$ such that $H\left(\vartheta_{1}, \omega_{e}, \omega_{p 1}\right)=0$ and

$$
\frac{\mathrm{d} H\left(\vartheta_{1}, \omega_{e}, \omega_{p 1}\right)}{\mathrm{d} \vartheta}<0 .
$$


Moreover,

$$
\frac{\mathrm{d} H\left(0, \omega_{e}, \omega_{p 1}\right)}{\mathrm{d} \vartheta}=0
$$

and, due to 20,

$$
\lim _{\vartheta \rightarrow \infty} \frac{\mathrm{d} H\left(0, \omega_{e}, \omega_{p 1}\right)}{\mathrm{d} \vartheta}=a_{p 1}^{\max }-a_{e}^{\max }<0 .
$$

Let us assume that the inequality $\mathrm{A} 2$ is violated, i.e., there exists $\vartheta_{2} \in\left[\vartheta_{p}, \vartheta_{0}\right]$ such that

$$
a_{p 1}^{\max }\left(1-\exp \left(-\frac{\vartheta_{2}}{\tau_{p 1}}\right)\right)-a_{e}^{\max }\left(1-\exp \left(-\frac{\vartheta_{2}}{\tau_{e}}\right)\right)>0 .
$$

Thus, due to A3-A6, there exist at least three non-negative distinct zeros of the function $\mathrm{d} H\left(\vartheta, \omega_{e}, \omega_{p 1}\right) / \mathrm{d} \vartheta$. Therefore, the second derivative

$$
\begin{aligned}
& \frac{\mathrm{d}^{2} H \vartheta, \omega_{e}, \omega_{p 1}}{\mathrm{~d} \vartheta^{2}} \\
& =\frac{a_{p 1}^{\max }}{\tau_{p 1}} \exp \left(-\frac{\vartheta}{\tau_{p 1}}\right)-\frac{a_{e}^{\max }}{\tau_{e}} \exp \left(-\frac{\vartheta}{\tau_{e}}\right),
\end{aligned}
$$

should have at least two non-negative distinct zeros. However, the function A7 can have no more than one non-negative zero. This contradiction proves that the inequality A2 holds for $\vartheta \in\left[\vartheta_{p}, \vartheta_{0}\right]$, which completes the proof of the lemma.

\section{Appendix B \\ Proof of Lemma 2}

In the proof of the lemma, we distinguish two cases: (i) $\vartheta_{0} \leq \vartheta_{c 1}$ and (ii) $\vartheta_{0}>\vartheta_{c 1}$. Let us start with the first case. In this case, $\mathcal{H}\left(0, \vartheta_{0}\right)$ satisfies the inequality (59), meaning that the point $\left(\vartheta_{0}, \mathcal{H}\left(0, \vartheta_{0}\right)\right)$ lies on the upper boundary of $C_{0}\left(\omega_{e}, \omega_{p 1}\right)$. Moreover, due to [53),

$$
\mathcal{H}\left(\vartheta_{0}, \vartheta_{0}\right)=\int_{0}^{\vartheta_{0}} H\left(\xi, \omega_{e}, \omega_{p 2}\right) \mathrm{d} \xi
$$

meaning that the point $\left(\vartheta_{0}, \mathcal{H}\left(\vartheta_{0}, \vartheta_{0}\right)\right)$ lies on the upper boundary of $C_{0}\left(\omega_{e}, \omega_{p 2}\right)$. On the other hand, since $\vartheta_{p}^{*}$ is the unique point of absolute maximum of $\mathcal{H}\left(\vartheta_{p}, \vartheta_{0}\right)$ on the interval $\vartheta_{p} \in\left[0, \vartheta_{0}\right]$, then $\mathcal{H}\left(\vartheta_{p}^{*}, \vartheta_{0}\right)>$ $\max \left\{\mathcal{H}\left(0, \vartheta_{0}\right), \mathcal{H}\left(\vartheta_{0}, \vartheta_{0}\right)\right\}$, which proves the lemma in the case (i). In the case (ii), similarly to the case (i), the point $\left(\vartheta_{0}, \mathcal{H}\left(\vartheta_{0}, \vartheta_{0}\right)\right.$ lies on the upper boundary of $C_{0}\left(\omega_{e}, \omega_{p 2}\right)$, and the point $\left(\vartheta_{0}, \mathcal{H}\left(\vartheta_{p}^{*}, \vartheta_{0}\right)\right)$ lies above it. On the other hand, since $\vartheta_{0}>\vartheta_{c 1}$, then the point $\left(\vartheta_{0}, \mathcal{H}\left(\vartheta_{p}^{*}, \vartheta_{0}\right)\right)$ lies outside of $\operatorname{clo}\left(C_{0}\left(\omega_{e}, \omega_{p 1}\right)\right)$. Thus, in the second case, the statement of the lemma also is valid.

Received: 28 January 2014

Revised: 29 May 2014 